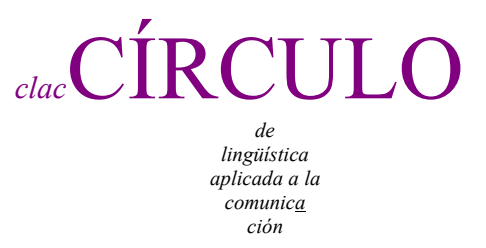

$68 / 2016$

\title{
EL BILINGÜISMO COMO PROTECCIÓN ANTE LA DEMENCIA: INCONSISTENCIAS EMPÍRICAS Y NUEVAS PROPUESTAS METODOLÓGICAS
}

\author{
Noelia Calvo, Laura Manoiloff, Edinson Muñoz, \\ Marcela Contreras, Agustín Ibáñez, Adolfo M. García \\ (información sobre los autores en página 37) \\ adolfomartingarcia en gmail com
}

\section{Resumen}

El deterioro cognitivo durante el envejecimiento normal o patológico puede retrasarse mediante experiencias que fomentan la reserva cognitiva. En este sentido, algunos estudios indican que el bilingüismo retrasaría los síntomas de la enfermedad de Alzheimer. Sin embargo, la evidencia se basa en diseños retrospectivos que arrojan resultados contradictorios. Este trabajo analiza dichos resultados, identifica las principales variables detrás de sus discrepancias y propone alternativas metodológicas. Primero, caracterizamos los posibles factores de confusión, a saber: criterios para definir el bilingüismo, diferencias en los diseños muestrales, instrumentos empleados para estudiar las habilidades cognitivas y variables que modulan el desempeño cognitivo. Luego proponemos que estas limitaciones se podrían evitar mediante diseños

Noelia Calvo, Laura Manoiloff, Edinson Muñoz, Marcela Contreras, Agustín Ibáñez, Adolfo M. García. 2016.

El bilingüismo como protección ante la demencia: inconsistencias empíricas y nuevas propuestas metodológicas.

Círculo de Lingüistica Aplicada a la Comunicación 68, 3-44.

http://www.ucm.es/info/circulo/68/calvo.pdf

http://revistas.ucm.es/index.php/CLAC

http://dx.doi.org/10.5209/CLAC.54521

(C) 2016 Noelia Calvo, Laura Manoiloff, Edinson Muñoz, Marcela Contreras, Agustín Ibáñez y Adolfo M. García. 
experimentales y el uso de instrumentos adecuados para controlar eficazmente dichas variables. Además, sugerimos incorporar tareas que ya han demostrado patrones predecibles de desempeño contrastivo entre individuos bilingües y monolingües (desventajas bilingües en el vocabulario, efectos nulos en ciertos componentes de la memoria de trabajo y ventajas en el control inhibitorio), y otras que podrían sumar datos muy valiosos (como las tareas de interferencia proactiva). Estas consideraciones pueden arrojar luz no sólo sobre la relación entre bilingüismo y reserva cognitiva, sino también sobre mecanismos más generales de compensación cognitiva.

Palabras clave: bilingüismo, enfermedad de Alzheimer, reserva cognitiva, diseño de investigación.

\section{Abstract}

Bilingualism and cognitive reserve: empirical inconsistencies and new methodological proposals. Cognitive decline throughout healthy or pathological aging can be slowed down by experiences which foster cognitive reserve. In this sense, some studies have suggested that bilingualism may delay the onset of Alzheimer's disease. However, the evidence stems from retrospective approaches yielding contradictory results. The present paper addresses these findings, identifies possible lurking variables, and outlines methodological alternatives thereof. First, we characterize possible confounding factors, namely: the criteria to establish bilingualism, differences in sample design, the instruments used to examine cognitive skills, and variables known to modulate life-long cognition. Second, we propose that these limitations could be largely circumvented through experimental approaches and the use of adequate instruments to measure such variables. Moreover, future research should incorporate tasks yielding predictable patterns of contrastive performance between bilinguals and monolinguals (bilingual disadvantages in vocabulary, null effects in working memory, advantages in inhibitory control and other executive functions), and other which could offer valuable insights (e. g., proactive interference tasks). Such considerations may shed light not just on the relationship between bilingualism and cognitive reserve, but also on more general mechanisms of cognitive compensation.

Keywords: bilingualism, Alzheimer's disease, cognitive reserve, research design. 
Índice

1. Introducción 5

2. La reserva cognitiva en bilingües con demencia: un corpus empírico inconsistente 7

3. Reparos metodológicos a los estudios 9

3.1. Limitaciones en la concepción y evaluación del bilingüismo 10

3.2. Variabilidad en el diseño muestral 11

3.3. Reparos sobre los instrumentos de evaluación y diagnóstico clínico 13

3.4. ¿Y si no es el bilingüismo? Otras variables ocultas que afectan la RC 15

4. La necesidad de mejoras metodológicas y enfoques experimentales 17

4.1. Mejoras en las herramientas para seleccionar y evaluar las muestras 17

4.2. Incorporación de tareas experimentales 19

4.3. Otras consideraciones 21

5. Conclusión 22

Referencias bibliográficas 23

Agradecimientos 37

Información sobre los autores 37

Anexo 38

1. Introducción

El término "bilingüismo" ha recibido diversas definiciones en la literatura especializada (García y otros, 2016). Mientras que algunos autores lo han empleado exclusivamente para referirse al dominio (cuasi) nativo de una segunda lengua (L2) (p. ej., Bloomfield, 1935), otros lo utilizan en referencia al uso alternado de ambas, sin importar el nivel de competencia (Mackey, 1968; Weinreich, 1953). En consonancia con esta última concepción, hoy suele concebirse al bilingüe a cualquier persona que utiliza dos lenguas o dialectos diariamente (Grosjean, 1994). Así, los bilingües se pueden clasificar según 
la edad de apropiación de la L2 (tempranos y tardíos), el nivel de competencia (incipiente, bajo, intermedio y alto) y la frecuencia de uso de la L2 (activos y latentes), entre otras variables. Dichas variables se asocian con perfiles neurocognitivos diferenciales. Por ejemplo, los correlatos anatómicos y funcionales de la lengua nativa (L1) y la L2 tienden a ser más similares en los bilingües tempranos que en los tardíos, en especial si la exposición a las L2 es constante (Ardal y otros, 1990; Gómez-Ruiz, 2010; Kim y otros, 1997; Neville y otros, 1992, 1997; Paradis, 2009; Perani y otros, 1996; Ullman, 2001; Weber-Fox y Neville, 1997). En suma, la población bilingüe se caracteriza por gran heterogeneidad sociodemográfica, lingüística y cognitiva.

Otra línea de investigación indica que el bilingüismo sostenido afecta múltiples funciones cognitivas. Los individuos bilingües presentan ventajas en ciertas funciones ejecutivas, como el control inhibitorio y determinados componentes de la memoria de trabajo (Ardila, 2012; Bialystok y otros, 2009; Calvo y otros, 2016; Michon, 2016). Estos efectos se han observado en niños (Adi-Japha y otros, 2010; Bialystok, 2011; Carlson y Meltzoff, 2008), adultos jóvenes (Costa y otros, 2008; Prior y MacWhinney, 2010; Rodriguez-Fornells y otros, 2006) y adultos mayores (Bialystok y otros, 2004; Salvatierra y Rosselli, 2010). Sin embargo, esta hipótesis también ha recibido varias críticas (ver Duñabeitia y Carreiras, 2015; García Pentón y otros, 2015).

Asimismo, se ha propuesto que el bilingüismo favorecería la reserva cognitiva (RC) (Bak y otros, 2014; Kavé y otros, 2008), a saber, la capacidad de compensación o resiliencia neurofuncional ante el daño cerebral o el envejecimiento (Cabeza y Dennis, 2013; Stern, 2012). En este sentido, se ha propuesto que diversas experiencias sociales y cognitivas retrasarían o reducirían el declive funcional que sobreviene a cualquier forma de deterioro neural (Stern, 2009; Cabeza y Dennis, 2013, Díaz-Urueta y otros, 2010).

Así, algunos estudios demostraron que los síntomas de la demencia (Alladi y otros, 2013; Bialystok y otros, 2007, 2014; Cherktow et al, 2010; Craik y otros, 2010; Woumans y otros, 2015) y del deterioro cognitivo leve (DCL) (Bialystok y otros, 2014; Ossher y otros, 2013) se presentan más tardíamente en los pacientes bilingües/plurilingües que en los monolingües. Además, Schweizer y otros (2012) documentaron un desempeño cognitivo comparable entre pacientes bilingües y monolingües con EA, a pesar del mayor deterioro cerebral en los primeros. Otros estudios sugieren que el bilingüismo sostenido se asocia positivamente con la integridad 
de la materia blanca (Gold y otros, 2013; Olsen y otros, 2015) y la densidad de la materia gris (Abutalebi y otros, 2014, 2015) en regiones críticas para las funciones ejecutivas.

Sin embargo, un análisis imparcial de la evidencia revela marcadas inconsistencias. Varios estudios no han podido replicar los resultados anteriores (Clare y otros, 2014; Crane y otros, 2010; Kowoll y otros, 2015; Lawton y otros, 2015; Sanders y otros, 2012; Zahodne y otros, 2014). Además, los estudios confirmatorios presentan varias debilidades metodológicas críticas. Finalmente, las diferencias volumétricas entre los cerebros bilingües y monolingües no implican directamente un aumento en la RC.

Es probable que el bilingüismo contribuya a la RC. Sin embargo, antes de abonar esta hipótesis es fundamental abordar los resultados contradictorios, identificar los posibles factores de confusión y esbozar alternativas metodológicas superadoras. Este trabajo persigue tales objetivos (véase Calvo y otros, 2015, y agradecimientos). Primero, revisamos la evidencia disponible y destacamos sus inconsistencias y diferencias metodológicas. Luego, caracterizamos los posibles factores de confusión que pueden haber influido en los resultados. Nuestro foco está puesto en los criterios para determinar el bilingüismo, las diferencias en el diseño muestral, los instrumentos de evaluación cognitiva, y el rol de otras variables que modulan la RC. Finalmente, proponemos que estas limitaciones se pueden minimizar mediante innovaciones en los instrumentos y los diseños empleados.

2. La reserva cognitiva en bilingües con demencia: un corpus empírico inconsistente Los principales argumentos a favor del rol neuroprotector del bilingüismo provienen de análisis retrospectivos de registros clínicos. Aunque algunos estudios recientes han explorado el tema considerando trastornos como la enfermedad de Parkinson (Hindle y otros, 2015), el principal corpus empírico surge de la comparación de pacientes monolingües y bilingües con EA. En un artículo pionero, Bialystok y otros (2007) observaron que la demencia se presentaba aproximadamente cuatros años más tarde en 184 inmigrantes bilingües que hablaban inglés y cualquier otra lengua. Craik y otros (2010) documentaron resultados similares en una muestra que incluía 211 inmigrantes y no inmigrantes. También se observó un retraso en la aparición de aproximadamente 4,5 años en 134 pacientes mayores con $\mathrm{EA}$, entre los que se incluían bilingües no 
inmigrantes (Woumans y otros, 2015). Este patrón se replicó en 648 sujetos con distintos tipos de demencia (Alladi y otros, 2013) y en 149 pacientes con DCL y EA (Bialystok y otros, 2014). Además, un estudio con 853 individuos sanos determinó que los bilingües mayores tuvieron un mejor desempeño cognitivo que el que se había pronosticado a partir de su estado cognitivo basal (Bak y otros, 2014). Sin embargo, otros estudios han apoyado la hipótesis sólo de manera parcial, mediante evidencia confirmatoria sólo para ciertos subgrupos. Cherktow y otros (2010) compararon la edad de inicio de los síntomas y del diagnóstico en 632 sujetos que eran pacientes monolingües, bilingües y plurilingües con EA. Los participantes incluían inmigrantes y no inmigrantes, con diferentes L1 y L2. Si bien se halló un retraso de tres años en los inmigrantes bilingües y plurilingües, no se observó ningún beneficio significativo en los bilingües no inmigrantes. Además, se reportó un efecto protector en los no inmigrantes cuya L1 era el francés, pero no en aquellos cuya L1 era el inglés. Ossher y otros (2013) estudiaron a 111 pacientes con DCL y observaron un retraso en la aparición de los síntomas sólo en los bilingües amnésicos. Asimismo, un estudio sobre funciones ejecutivas (inhibición, atención y memoria de trabajo) en participantes mayores sanos arrojó ventajas sólo en individuos con alto nivel de competencia en L2 (Gollan y otros, 2011). Por su parte, Kousaie y Phillips (2012) administraron una tarea de Stroop en una muestra de 118 adultos jóvenes y mayores, monolingües y bilingües; sólo los adultos jóvenes presentaron una ventaja de velocidad de procesamiento en relación con sus contrapartes monolingües, pero esto no se asoció con disminuciones de la interferencia. El campo se complejiza aún más al considerarse varios estudios longitudinales sobre la EA que no encontraron mayor RC en individuos bilingües. En el trabajo de Crane y otros (2010), se evaluó la demencia en 2520 japoneses-estadounidenses de segunda generación (que no presentaban la enfermedad en la evaluación basal), en tres ocasiones durante seis años. El uso de japonés hablado y escrito en la mediana edad no se relacionó con las tasas de deterioro en etapas posteriores. Otro estudio (Sanders y otros, 2012) comparó la incidencia de demencia en 1779 usuarios de inglés mayores, nativos y no nativos. El segundo grupo no mostró evidencia de una mayor RC; de hecho, presentó un pequeño aumento (aunque no significativo) en el riesgo de padecer demencia. En particular, los hablantes no nativos con al menos 16 años de educación aumentaron cuatro veces el riesgo de demencia en comparación con los participantes con menos 
educación. En el mismo sentido, Zahodne y otros (2014) evaluaron a 1067 pacientes con EA en intervalos de 18-24 meses durante 23 años. Alrededor de 300 sujetos presentaron demencia en el transcurso del estudio. En la evaluación inicial, el bilingüismo se había asociado con mejor rendimiento mnésico y ejecutivo. Sin embargo, esto no se relacionó con tasas de deterioro cognitivo o conversión a demencia. Finalmente, Kowoll y otros (2015) no observaron diferencias significativas en pruebas neuropsicológicas entre pacientes monolingües y bilingües con DCL/EA en una muestra de 86 participantes. Sin embargo, los autores concluyeron que la lengua dominante puede verse comprometida primero en pacientes bilingües con DCL, mientras que los déficits en la lengua no dominante aparecían más tarde, con la manifestación de la EA.

También se han informado resultados nulos en estudios de cohortes. Clare y otros (2014) realizaron una investigación transversal en 86 pacientes con EA temprana. Al momento del diagnóstico, los bilingües tenían en promedio tres años más que los monolingües, pero también presentaban déficits cognitivos significativamente mayores. Además de tener un desempeño relativamente mejor en tareas de conflicto de respuesta e inhibición, los bilingües no presentaron ventajas significativas en la función ejecutiva. Por su parte, Lawton y otros (2015) evaluaron a 1789 hispano-estadounidenses de más de 60 años (la mitad de los cuales eran inmigrantes), cada 12 a 15 meses durante 10 años. A 55 participantes se les diagnosticó EA y a 26, demencia vascular. Cabe mencionar que la media de edad de diagnóstico no tenía una diferencia significativa entre los pacientes bilingües y los monolingües (nacidos en Estados Unidos o inmigrantes). Para obtener mayores detalles sobre estos estudios, ver la Tabla 1.

En resumen, la literatura comprende tantos resultados positivos como nulos. Esto indica que el bilingüismo podría contribuir a la RC, pero sólo en ciertas condiciones aun desconocidas. Estas discrepancias pueden reflejar, en gran medida, las diferencias metodológicas entre los estudios disponibles y deficiencias en su realización. A continuación se analizan estos factores a fin de promover aproximaciones más robustas en el campo.

\section{Reparos metodológicos a los estudios}

La investigación no experimental enfrenta múltiples desafíos cuando se estudia a una población tan diversa como los bilingües, en especial aquellos afectados por 
enfermedades tan heterogéneas y complejas como la EA. La imposibilidad de controlar factores fundamentales y de recolectar datos pertinentes de los sujetos lleva a una variabilidad generalizada en y entre los grupos. En este sentido, los estudios anteriores presentan diferencias y/o limitaciones importantes en relación con cuatro factores: (i) la concepción de bilingüismo y la evaluación de la competencia lingüística, (ii) el diseño muestral, (iii) los instrumentos usados para evaluar las habilidades cognitivas y diagnosticar el cuadro clínico subyacente, y (iv) el estudio de otras variables que afectan la RC.

\subsection{Limitaciones en la concepción y evaluación del bilingüismo}

La literatura presenta varios reparos con respecto a la concepción de bilingüismo y a la evaluación de la competencia lingüística. La mayoría de los estudios retrospectivos en la sección 2 establecieron el bilingüismo y la competencia lingüística mediante entrevistas subjetivas con los pacientes (Bialystok y otros, 2007; Cherktow y otros, 2010; Craik y otros, 2010; Crane y otros, 2010; Sanders y otros, 2012; Woumans y otros, 2015) o con sus cuidadores (Cherktow y otros, 2010; Craik y otros, 2010; Schweizer y otros, 2012; Alladi y otros, 2013; Woumans y otros, 2015). Sin embargo, la estimación subjetiva de la competencia puede ser poco confiable y verse sesgada por percepciones personales (Hulstijn, 2012). Más aun, algunos estudios (por ejemplo, Cherktow y otros, 2012) carecen de datos sobre la adquisición de la L2 y la situación migratoria de los participantes y estimaron esta información en base a impresiones personales.

Del mismo modo, la edad de adquisición de la L2 varía enormemente entre los estudios y dentro de los mismos. Por ejemplo, en el caso de Woumans y otros (2015), algunos participantes habían adquirido la L2 desde su nacimiento; otros, cerca de la pubertad; y otros, durante la adultez. Esto constituye otro posible factor de confusión, ya que los bilingües recurren a diferentes mecanismos cognitivos según la forma y la edad de apropiación de la L2, como la adquisición incidental en comparación con el aprendizaje metalingüístico (Paradis, 2009), además de las circunstancias socioculturales que enmarcan el desarrollo bilingüe, como el bilingüismo circunstancial en comparación 
con el bilingüismo por elección en inmigrantes y estudiantes de L2, respectivamente (Valdés y Figueroa, 1994).

Además, la mayoría de los estudios confunden bilingüismo con plurilingüismo. Por ejemplo, en el estudio realizado por Alladi y otros (2013), el 26,2\% de los participantes hablaba dos lenguas, mientras que el 34\% hablaba tres o más. Esta consideración no es trivial, pues los recursos neurocognitivos implicados durante el procesamiento bilingüe son sensibles a la presencia de otras lenguas (Marian y otros, 2013). Es más: una comparación entre bilingües, trilingües y plurilingües (Kave y otros, 2008) demostró que el número de lenguas no nativas que maneja un individuo influye en el desempeño cognitivo, más allá del efecto de las variables demográficas.

Por otra parte, la mayoría de los estudios incluye bilingües que hablaban distintos pares de lenguas, en los que sólo algunas eran similares tipológicamente (por ejemplo, Kowoll y otros, 2015; Woumans y otros, 2015). Sin embargo, los mecanismos de acceso y de control en el procesamiento bilingüe difieren según la distancia tipológica entre las lenguas (García, 2014b; Tao y otros, 2011). Además, la mayoría de los estudios carece de evaluaciones para determinar el monolingüismo, lo que constituye un aspecto fundamental para la literatura sobre bilingüismo y RC, pues puede que no exista algo así como un "monolingüe puro" (De Bot $y$ Jaensch, 2015).

Después de todo, la literatura sobre bilingüismo y $\mathrm{RC}$ es inconsistente $\mathrm{y}$, a veces, presenta imperfecciones en su caracterización de esta primera variable. Esto se debe, en gran medida, a la adopción de enfoques retrospectivos no experimentales, que excluyen la construcción de muestras bien controladas. Así, es indispensable desarrollar procedimientos de muestreo más consistentes, en especial de cara a la exploración de posibles correlatos neurológicos. Es más, la competencia en L2 se correlaciona positivamente con el volumen de materia gris en áreas críticas para el control cognitivo (Stein y otros, 2012) y con la edad de diagnóstico y de la aparición de los síntomas de la EA (Gollan y otros, 2011).

\subsection{Variabilidad en el diseño muestral}

Los diseños retrospectivos dificultan la aleatorización de las muestras, cuyos tamaños varían considerablemente entre los estudios. En esta revisión, tres estudios incluyeron 
menos de 50 participantes (Gollan y otros, 2011; Kowoll y otros, 2015; Schweizer y otros, 2012) ; dos, menos de 100 (Clare y otros, 2014; Lawton y otros, 2015) ; seis, menos de 250 (Bialystok y otros, 2007, 2014; Craik y otros, 2010; Kousaie y Phillips, 2012; Ossher y otros, 2013; Woumans y otros, 2015) ; tres, entre 500 y 1000 (Alladi y otros, 2013; Bak y otros, 2014; Cherktow y otros, 2010); y otros tres, entre 1000 y 2500 (Crane y otros, 2010; Sanders y otros, 2012; Zahodne y otros, 2014). Si bien el tamaño de los efectos se relaciona parcialmente con el tamaño de la muestra, en especial cuando los efectos son consistentes (Little, 2013), estas diferencias entre los estudios podrían explicar parte de sus discrepancias.

Tanto en los estudios retrospectivos como en los diseños experimentales, las variables demográficas (como edad, sexo, educación) y las variables clínicas (por ejemplo, otras patologías) se deben emparejar entre los grupos comparados para ajustar los datos en caso de que haya posibles factores de confusión y aumentar la precisión del análisis. En este sentido, otro reparo a los estudios es que comparan muestras de diferente tamaño. En algunos casos, había 50\% más de monolingües que de bilingües (por ejemplo, Cherktow y otros, 2010; Lawton y otros, 2015; Zahodne y otros, 2014) y en el estudio realizado por Sanders y otros (2012), uno de los grupos triplicaba al otro en tamaño. Las muestras con tanta diferencia en tamaño pueden sesgar los resultados, ya que el grupo más grande podría presentar una varianza mayor y, por lo tanto, ser más representativo de su población correspondiente. Esto es especialmente cierto cuando el grupo más pequeño es el que está compuesto por bilingües, pues su selección aleatorizada podría no representar de manera realista el amplio espectro de edades de adquisición, niveles de competencia y grados de exposición a la L2.

Este escenario se complica aún más por la heterogeneidad de las muestras. Algunos estudios compararon monolingües y bilingües, otros examinaron diferentes tipos de bilingües y otros consideraron diferentes tipos de demencia, a veces sin un grupo control (ver más detalles en la Tabla 1). En particular, las demencias son enfermedades heterogéneas y pueden diferir en la susceptibilidad de diagnóstico temprano, velocidad de la progresión, grado de compromiso o vulnerabilidad genética y la naturaleza local o global de los dominios cognitivos afectados. Además, la mayoría de los estudios no explicitó los criterios de inclusión y exclusión. Finalmente, estos estudios no complementaron los cálculos de significatividad (valor $p$ ) con cálculos del tamaño del 
efecto. Esto sería de utilidad para determinar el número mínimo de participantes requerido para evitar errores tipo II o $\beta$ (Sullivan y Feinn, 2012). Es importante mencionar que el tamaño de la muestra se debe establecer antes de iniciar cualquier estudio y, dentro de lo posible, no se debe cambiar durante el curso del mismo (Kadam y Bhalerao, 2010). En este sentido, sólo unos pocos estudios contemplaron estas mediciones (Bak y otros, 2014; Bialystok y otros, 2014; Clare y otros, 2014, Woumans y otros, 2015) y algunos excluyeron participantes que no eran pertinentes para el análisis, con lo que cambiaron el tamaño de la muestra inicial (Cherktow y otros, 2010; Crane y otros, 2010; Gollan y otros, 2011; Lawton y otros, 2015; Sanders y otros, 2012; Zahodne y otros, 2014).

Para resumir, la literatura presenta gran variabilidad en el cálculo y la estabilidad del tamaño muestral. Además, algunos aspectos estadísticos fundamentales no se han explicitado lo suficiente. Es posible que los estudios retrospectivos carezcan de potencia para ser significativos, lo cual redobla la necesidad de contemplar estos aspectos (Sullivan y Feinn, 2012).

\subsection{Reparos sobre los instrumentos de evaluación y diagnóstico clínico}

El diagnóstico del DCL y de la EA puede basarse en criterios muy variables. Los catorce estudios (excepto uno) que tenían como objetivo estudiar grupos con patologías establecieron el diagnóstico mediante el consenso de personal clínico calificado. En siete de ellos (Bialystok y otros, 2007, 2014; Cherktow et al, 2010; Craik y otros, 2010; Kowoll y otros, 2015; Lawton y otros, 2015; Sanders y otros, 2012), la EA se diagnosticó siguiendo los criterios NINCDS-ADRDA (McKhann y otros, 1984); dos siguieron los criterios del DSM III y IV (Crane y otros, 2014; Sanders y otros, 2012, respectivamente) ; uno usó el manualCIE-10 (Clare y otros, 2014); y tres usaron otros criterios (Alladi y otros, 2013; Clare y otros, 2014; Schweizer y otros, 2012), en ausencia del consenso de un equipo clínico.

Además de estas discrepancias, la patología subyacente no fue consistente entre los estudios ni dentro de ellos. Ossher y otros (2013) se enfocaron en pacientes con DCL, según los criterios en Petersen (2004). Bialystok y otros (2014) consideraron esta enfermedad en conjunto con la EA, siguiendo los criterios de diagnóstico propuestos 
por Albert y otros (2011). En el caso de los 130 pacientes del estudio realizado por Cherktow y otros (2010), la aparición de la demencia se definió como la visita clínica en la cual se cambió un diagnóstico previo de DCL a EA. Además, el estudio de Bialystok y otros (2007) incluyó a 52 pacientes a quienes se les diagnosticó otras demencias (entre las que se incluía posible EA). Estas diferencias plantean más dudas sobre la consistencia de los resultados, dado que el diagnóstico puede variar considerablemente según el tipo de discapacidad que presenten los pacientes y los criterios empleados para establecer el cuadro clínico subyacente (Burvill, 1993).

Otros reparos se refieren a los instrumentos empleados para evaluar la demencia. Prácticamente todos los estudios usaron el MMSE (Folstein y McHugh, 1975). Aunque otros han incorporado medidas adicionales, como la Evaluación Neurológica Conductual (BNA) (Bialystok y otros, 2014; Schweizer y otros, 2012), la Clasificación Clínica de la Demencia (CDR) (Alladi y otros, 2013; Schweizer y otros, 2012) y el Examen Cognitivo de Addenbrooke-Revisado (ACE-R) (Alladi y otros, 2013), en la mayoría de los casos el MMSE fue la única medición neuropsicológica empleada (por ejemplo, Bialystok y otros, 2007; Cherktow y otros, 2010; Craik y otros, 2010; Kowoll y otros, 2015; Woumans y otros, 2015). Sin embargo, muchas investigaciones muestran que el MMSE tiene un valor limitado para diagnosticar la aparición de la demencia (Kim y Caine, 2014; Wind y otros, 1997), medir su progresión en periodos inferiores a tres años (Clark y otros, 1999), detectar el DCL (Tang-Wai y otros, 2003) e incluso evaluar el perfil cognitivo general (Feher y otros, 1992). En la actualidad, es posible padecer de la EA y aun así obtener un puntaje de 30/30 en esta prueba (Shirosky y otros, 2007). Además, los síntomas iniciales de EA podrían ser incluso más difíciles de detectar en individuos con una RC mayor.

Es más, ni el MMSE ni ninguno de los otros instrumentos usados están específicamente diseñados para evaluar los dominios ejecutivos, cuya evaluación puede ser fundamental para determinar el impacto específico del bilingüismo en la RC (Bialystok y otros, 2014). Adviértase también que la disfunción ejecutiva puede constituir una de las manifestaciones tempranas de la EA (Grober y otros, 2008). Así, la inclusión de mediciones adicionales sensibles (en especial, aquellas que indaguen sobre el desempeño ejecutivo) podría ser esencial para el progreso del campo. 


\section{4. ¿Y si no es el bilingüismo? Otras variables ocultas que afectan la RC}

Para que pueda establecerse una relación específica entre el bilingüismo y la $\mathrm{RC}$ debe excluirse la influencia de factores de confusión. Los estudios disponibles sólo han alcanzado esta meta de modo parcial. Mientras que la mayoría de ellos incluyen datos sobre la edad, el nivel educacional y el nivel socioeconómico del paciente, rara vez contemplan otros factores pertinentes.

La RC se puede aumentar a través de diferentes hábitos y rasgos de personalidad. Por ejemplo, se puede potenciar a través de las relaciones sociales (Bennett y otros, 2006) o la estimulación intelectual constante (más que la educación) (Scarmeas y Stern, 2004). Otros factores que contribuyen son el buen estado físico general, la cantidad de ejercicio y el tipo de actividad física permanente (ver, por ejemplo, Davenport y otros, 2012). En particular, el ejercicio aeróbico saludable reduce la pérdida de volumen hipocampal y mejora la memoria en la adultez (Erickson y otros, 2011). La RC también puede mejorarse mediante el desarrollo de habilidades emocionales que favorecen el comportamiento adaptativo y la resiliencia ante el estrés (Staudinger y otros, 1993). Estas habilidades pueden aparecer preponderantemente en individuos inmigrantes; por consiguiente, las conclusiones de algunos estudios (como Bialystok y otros, 2007; Cherktow y otros, 2011; Craik y otros, 2010; Kowoll y otros, 2015) podrían implicar atribuciones erróneas o generalizaciones excesivas, de modo que podrían no ser aplicables a cualquier tipo de bilingüe.

Por otro lado, algunos factores no controlados adecuadamente pueden ir en detrimento de la RC. Algunos pueden tener un rol distintivo en las poblaciones bilingües, sobre todo en grupos de inmigrantes. Uno de los factores que más inciden sobre la RC es el nivel socioeconómico. Dicho factor se ha analizado en la mayoría de los estudios con grupos de inmigrantes bilingües. Esta variable se ha relacionado con una reducción del riesgo de presentar DCL o EA (Sattler y otros, 2012); sin embargo, la migración frecuente involucra una pérdida del nivel socioeconómico y un aumento de las tasas de enfermedad mental (Bhugra y Becker, 2005).

Además, las poblaciones inmigrantes presentan un aumento en el riesgo de trastornos neurológicos congénitos o adquiridos (White y otros, 2005; Zahuranec y otros, 2006), abuso de alcohol producto del estrés por la aculturación (Caetano, 2006; Szaflarski y 
otros, 2011), trastornos alimentarios (Bulik, 2006; Geller y otros, 1999), mala calidad de sueño (Voss y Tuin, 2008) y la adquisición de hábitos dañinos para la salud, como el tabaquismo (Bethel y otros, 2005). Al mismo tiempo, el uso sostenido de más de una lengua se ha relacionado con mayores posibilidades de consumo de alcohol en la adolescencia (Epstein y otros, 1996) y con un menor reconocimiento de los peligros asociados al tabaquismo (Unger y otros, 2000). Las diferencias entre bilingües y monolingües en estas variables pueden ser la base de las discrepancias en la literatura. La neurogénesis en el hipocampo se reduce en el caso de los bebedores excesivos (Stevenson y otros, 2009). A su vez, el consumo moderado de alcohol puede favorecer la liberación de acetilcolina en el hipocampo (Henn y otros, 1998) y, por lo tanto, reducir el riesgo de EA. También el consumo de cigarrillo acelera el adelgazamiento cortical, un biomarcador confiable de deterioro cognitivo (Karama y otros, 2015). Los trastornos de memoria también se asocian con otros hábitos no saludables, como la falta de sueño o trastornos alimentarios (Walker y Stickgold, 2006; Green y Rogers, 1998), entre otros.

Sin embargo, estos factores casi no se han controlado en la literatura revisada. Algunos de ellos se han considerado con grados de confiabilidad variables. Los datos más objetivos fueron proporcionados por Schweizer y otros (2012), quien aplicó el Índice de Katz de independencia en las actividades de la vida diaria. Bialystok y otros (2014) utilizaron un cuestionario de autoevaluación para recoger datos de los pacientes con respecto a su dieta, consumo de alcohol, hábito de fumar y actividad física y social. Sin embargo, este enfoque es menos confiable debido a los sesgos inherentes al informar sobre prácticas reprobadas socialmente. Por su parte, Alladi y otros (2013) entrevistaron a familiares de los pacientes para recopilar información sobre factores de riesgo vasculares, diabetes, tabaquismo y alcoholismo (aunque el estudio no ofrece mayores especificaciones metodológicas sobre la recolección de datos). Los resultados también pueden haberse visto sesgados por el uso de una muestra heterogénea de individuos bilingües mayores con un nivel educativo muy alto. De hecho, el desarrollo de la enfermedad puede ser más lento y más sensible a otros factores (distintos del bilingüismo) en los participantes mayores que en los más jóvenes. Por añadidura, ninguno de estos estudios parece haber empleado instrumentos diseñados específicamente para evaluar dichas variables (ver sección 4. 1). 
En resumen, la escasez de información sobre estos factores debilita los argumentos a favor y en contra de una relación positiva entre el bilingüismo y la RC. Como proponemos a continuación, los estudios futuros deberían considerar más cuidadosamente otras variables que puedan promover cambios plásticos positivos (por ejemplo, fortalecimiento sináptico) o negativos (por ejemplo, atrofia o debilitamiento sináptico) que puedan estar relacionados con DCL y EA y que puedan tener efectos importantes en la población bilingüe.

\section{La necesidad de mejoras metodológicas y enfoques experimentales}

La evidencia de la relación entre el bilingüismo y la $\mathrm{RC}$ es inconsistente y abunda en limitaciones metodológicas. Sin embargo, estas deficiencias no implican necesariamente que la RC no se vea afectada por la experiencia bilingüe. El tema podría abordarse más adecuadamente mejorando el control de las variables muestrales e incorporando tareas experimentales.

\subsection{Mejoras en las herramientas para seleccionar y evaluar las muestras}

Muchas de estas limitaciones se pueden rodear adoptando mejores instrumentos para evaluar y clasificar a los participantes, a fin de maximizar la homogeneidad interna de las muestras y la comparabilidad entre ellas. En primer lugar, se debe considerar cuidadosamente la evaluación del bilingüismo y de la competencia bilingüe. Los cuestionarios deben ser lo suficientemente completos para evaluar información fundamental, como la información biográfica de los participantes y sus familiares, uso de la L2, nivel de competencia, dominancia lingüística, edad de apropiación de la L2, actitud y preferencia lingüísticas (Codo, 2008). Además, para maximizar la comparabilidad entre los estudios, es recomendable emplear cuestionarios estandarizados. Una buena posibilidad es el Language History Questionnaire 2.0 (Li y otros, 2014). También, para lograr un mejor control de la competencia en L2, sería útil incluir medidas objetivas, como pruebas de idiomas estandarizadas o exámenes en lenguas específicas (como DIALANG, Diplômes d’Études en Langue Française, Zertifikar Deutsch, Certificado de Español Lengua y Uso), pruebas de vocabulario 
como LLEX (Meara, 1994), tests de compleción (Hulstijn, 2010) o incluso el Test de la afasia para bilingües (Paradis and Lecours, 1979; Paradis 2011).

En segundo lugar, los factores de confusión enumerados en la sección 3. 4 podrían controlarse de manera más efectiva utilizando instrumentos que los evalúen directamente. Por ejemplo, la Entrevista diagnóstica internacional compuesta (Robins y otros, 1989) evalúa los hábitos de consumo de alcohol, tabaco y drogas. También considera la calidad, gravedad y trayectoria de la dependencia de drogas, a la vez que proporciona información valiosa sobre posibles discapacidades y trastornos mentales comórbidos. De manera similar, el Inventario de trastornos del sueño (Zammit y otros, 1999) ha demostrado su utilidad en la investigación de la EA. Este instrumento evalúa una amplia variedad de comportamientos del sueño, al igual que la frecuencia y gravedad de sus alteraciones y la carga que significa para los cuidadores (Tractenberg y otros, 2006). También se pueden recolectar datos pertinentes a través del Cuestionario de trastornos de la conducta alimentaria (Fairburn y Beglin, 1994). Debido a que estas herramientas pueden ayudar a esclarecer el rol de las variables ocultas en los efectos observados, los estudios futuros deben incluir, al menos, sus versiones abreviadas. Asimismo, según la propuesta de Hogervorst y otros (2008), los pacientes con deterioro cognitivo deberían completar una evaluación de hipotiroidismo, ya que esta enfermedad se correlaciona con un menor desempeño en el MMSE basal, independiente de los factores cardiovasculares, la evaluación de FT4, la edad, el sexo, la educación y el estado de ánimo. Además, puesto que los inmigrantes presentan un riesgo mayor de varios trastornos (Bulik, 2006; Caetano, 2006; Geller y otros, 1999; Szaflarski y otros, 2011; Voss y Tuin, 2008; White y otros, 2005; Zahuranec y otros, 2006), debería priorizarse a las poblaciones de no inmigrantes para establecer relaciones más claras entre el bilingüismo y la RC (Fuller Thomson y Kuh, 2014).

En tercer lugar, se deben incorporar indicadores más sensibles de evaluación cognitiva general para complementar el MMSE. Se pueden obtener evaluaciones confiables mediante el uso de la Escala de demencia de Mattis (Mattis, 1998), la Escala de evaluación de la enfermedad de Alzheimer (Mohs, 1994; Mohs y Cohen, 1988; Mohs y otros, 1983) y la Evaluación cognitiva de Montreal (Nasreddine y otros, 2005). Este último instrumento, en particular, es breve, cuenta con propiedades psicométricas 
confiables (Dalrymple-Alford y otros, 2010) y detecta de manera efectiva el DCL y déficits cognitivos sutiles (Hoops y otros, 2009).

Además, como señalan Bialystok y otros (2014), las evaluaciones amplias del estado cognitivo deben complementarse con mediciones sensibles de funciones ejecutivas, puesto que el bilingüismo modula tales dominios. Una buena opción sería la batería de evaluación frontal INECO (Torralva y otros, 2009a), una herramienta abreviada para evaluar las enfermedades neurodegenerativas (Gleichgerrcht y otros, 2011; Torralva y otros, 2009a), en general, y las funciones ejecutivas frontales (Roca y otros, 2011), en particular. Sobre un puntaje máximo de 30 puntos, un puntaje de corte de 25 puntos ha demostrado una sensibilidad de 96,2\% y una especificidad de $91,5 \%$ en la detección de pacientes con síndrome disejecutivo (Torralva y otros, 2009b). Su aplicación puede ser especialmente informativa ya que la RC puede ser modulada por las habilidades ejecutivas tanto en la EA como en el DCL (Buckner, 2004).

En síntesis, la relación entre el bilingüismo y la RC se puede explorar más claramente mediante la incorporación de mediciones confiables de competencia bilingüe, estilo de vida, consumo de drogas, estado cognitivo y funcionamiento ejecutivo. Estas mediciones serían particularmente útiles en estudios con muestras intencionales y, además, podrían arrojar datos cruciales para explorar las relaciones con el desempeño de los pacientes en tareas experimentales, según lo descrito anteriormente.

\subsection{Incorporación de tareas experimentales}

Además de mejorar las herramientas que se utilizan en la investigación no experimental, el campo podría beneficiarse con la incorporación de experimentos controlados, sobre todo mediante tareas sensibles al bilingüismo. Diferentes paradigmas arrojan resultados característicos de los bilingües en relación con los monolingües: desventajas en el procesamiento verbal, efectos nulos en ciertos componentes de la memoria de trabajo y ventajas en otras funciones ejecutivas (Bialystok y otros, 2009, 2012). Por consiguiente, si los individuos bilingües tienen una mayor RC, estas tareas deben producir patrones de desempeño predecibles cuando se usan en muestras de individuos con demencia.

Primero, a lo largo de la vida, los bilingües muestran desventajas en tareas verbales realizadas en una sola lengua (Bialystok, 2009). Esto se ha demostrado 
sistemáticamente a través de Test de vocabulario en imágenes Peabody (Dunn y Dunn, 1997), en el que los participantes deben decidir cuál de cuatro imágenes corresponde al sustantivo enunciado por el investigador. Tanto en niños como en adultos, los monolingües tienen mejor desempeño que los bilingües en esta prueba (Bialystok, 2009). Así, si el bilingüismo aumenta la RC, se debería esperar que estas desventajas fueran menores o desaparecieran en tareas desarrolladas por muestras de individuos con demencia.

Segundo, hay otros dominios que parecen no verse alterados por los efectos del bilingüismo (Bialystok, 2009). Por ejemplo, el desempeño general en tareas de memoria de trabajo parece similar tanto en individuos bilingües como monolingües. Este resultado es especialmente consistente en tareas de span verbal (Bialystok y otros, 2008; Bonifacci y otros, 2010; Engel de Abreu, 2011; Feng, 2009; Namazi y Thordardottir, 2010). Por consiguiente, sería interesante explorar si este patrón se torna en una ventaja bilingüe cuando se comparan muestras de pacientes con EA.

Tercero, el bilingüismo mejora el control inhibitorio en tareas no verbales. Varios estudios que han usado una tarea Simon o una tarea Stroop han mostrado que los bilingües se desempeñan mejor que los monolingües de la misma edad (Bialystok y otros, 2004, 2008). Además, los bilingües parecen tener una conectividad funcional intrínseca más fuerte en la red de control frontoparietal y la red por defecto (default mode network), lo que podría ser beneficioso durante el envejecimiento (Grady y otros, 2015). Por añadidura, el desempeño en pruebas de función ejecutiva se reduce más rápidamente entre dos y tres años antes del diagnóstico de la EA (Grober y otros, 2008). Así, mediante investigaciones longitudinales con pacientes con EA, se podría estudiar si los subdominios ejecutivos (como las habilidades inhibitorias), en relación con otros dominios, se ven menos afectados por la progresión de la enfermedad en los individuos bilingües.

Finalmente, algunas tareas que no se usan con frecuencia en el campo del bilingüismo también pueden arrojar luz sobre el tema. Consideremos, por ejemplo, los paradigmas de interferencia proactiva (IP). La IP se refiere al efecto disruptivo de la información previa sobre la recuperación de información más reciente (Lustig y Hasher, 2002). Es decir, la IP se produce cuando la información almacenada en la memoria de largo plazo interfiere proactivamente con la información adquirida recientemente. La resolución de 
la IP involucra mecanismos de control proactivos y reactivos, que parecen verse robustecidos en individuos bilingües (Morales y otros, 2013a; Morales y otros, 2013b). En el envejecimiento normal, las habilidades de IP son sensibles a la edad y del deterioro cognitivo (Bowles y otros, 2003; Lustig y otros, 2001) y también pueden verse disminuidas en las etapas iniciales de la EA (Ebert y otros, 2009) y del DCL amnésico (Crocco y otros, 2014). Cabe mencionar que los sujetos pueden presentar deterioro cognitivo varios años antes de que se les diagnostique la EA (Amieva y otros, 2005).

\subsection{Otras consideraciones}

Para evaluar la relación entre bilingüismo y RC de modo más robusto también deberán considerarse otros aspectos. En primer lugar, se deberían fomentar enfoques más homogéneos para el diagnóstico de la EA. Hasta ahora, sólo algunos estudios han considerado las diferencias clinimétricas y culturales de los participantes. Los estudios futuros deberían contemplar ambos aspectos. Además, sería esencial combinar paradigmas conductuales con diferentes técnicas que revelen posibles biomarcadores de la EA a nivel genético, anatómico y de conectividad de redes. Primero, es importante mencionar que los genes de riesgo (como APOE y SORL1) y los genes causativos (APP, PSEN1 y PSEN2) de la EA se han expandido a más de 20 loci de riesgo (como ABCA7, BIN1, CD33, CD2AP, CLU, CR1, EPHA1, MS4A4E/MS4A6A y PICALM) (Karch y otros, 2014). Los cambios patológicos provocados por factores genéticos se podrían entender mejor utilizando biomarcadores de líquido cefalorraquídeo (Craft y otros, 2013; Ghidoni y otros, 2012; Kovacs y otros, 2010; Zhang y otros, 2005). Un desafío para este campo es explorar las correlaciones entre estos factores y los patrones neurodegenerativos diferenciales entre pacientes con EA bilingües y monolingües.

Por otra parte, la evidencia de neuroimágenes indica que la EA se caracteriza por alteraciones en la red por defecto (Sheline y otros, 2013), que presenta una conectividad funcional intrínseca mayor en los individuos bilingües que en los monolingües (Grady y otros, 2015). Nótese que el control proactivo se ha relacionado con la conectividad frontoparietal Theta (Cooper y otros, 2015) y que los individuos bilingües poseen una mejor resolución de los mecanismos de control (Morales y otros, 2013a, 2013b). Entonces, sería de utilidad evaluar si este patrón diferencial se mantiene al compararse 
pacientes con EA de ambos grupos. Además, el foco de la investigación debería ir más allá de la detección del retraso en la aparición de los síntomas de la EA. Sería beneficioso evaluar el impacto del bilingüismo en la progresión de la enfermedad y de otros déficits cognitivos asociados. Mientras que las mediciones tradicionales como el MMSE son ciegos a estos cambios, las tareas experimentales pueden revelar patrones progresivos en el curso de la enfermedad.

De este modo, se podrían obtener marcadores de neuroimagen o electrofisiológicos durante tareas activas para ayudar en el monitoreo progresivo de la detección de cambios cognitivos relacionados con el envejecimiento normal, el DCL y la EA. Por ejemplo, las tareas que requieren de la memoria a corto plazo, como aquellas que involucran el proceso de binding (Parra y otros, 2009, 2010), han demostrado ser sensibles a la EA temprana e incluso preclínica. Este sería un paradigma especialmente revelador para monitorear la aparición y la progresión de la EA temprana, puesto que dicho proceso no se ve afectado por el envejecimiento normal (Brockmole y otros, 2008, 2013; Brown y otros, 2010; Parra y otros, 2009). Además, los paradigmas de IP pueden mostrar variaciones en la memoria y en la $\mathrm{RC}$ tanto en el envejecimiento normal como en el curso de la EA. El desempeño de la IP disminuye durante el envejecimiento saludable (Bowles y otros, 2003; Lustig y otros, 2001), el DCL (Crocco y otros, 2014) y la EA (Ebert y otros, 2009). Así, se podrían generar nuevos hallazgos al comparar los biomarcadores de estas tareas en los individuos bilingües y monolingües con DCL o EA.

Por último, el campo debe expandir sus horizontes más allá del estudio de la EA y evaluar la $\mathrm{RC}$ en individuos bilingües que presenten otros trastornos. En este sentido, sería interesante explorar otras enfermedades que se caractericen tanto por síntomas lingüísticos (por ejemplo, la afasia progresiva primaria) como no lingüísticos (como la esclerosis lateral amiotrófica y la demencia frontotemporal en su variante conductual). Con esto se allanaría el camino para alcanzar conocimientos más refinados sobre el posible impacto del bilingüismo en la RC específica para algunos dominios.

\section{Conclusión}

La investigación de la RC en individuos bilingües ha demostrado ser muy desafiante debido a las múltiples variables que involucra. Las limitaciones que subyacen a las 
inconsistencias en todos los estudios se podrían evitar, en gran medida, a través de enfoques experimentales y un control más estricto de las variables pertinentes. Además, el campo podría ampliarse mediante enfoques que exploren no sólo el retraso en la aparición de la EA en bilingües, sino también los cambios que se producen durante el curso de la enfermedad. Estas consideraciones podrían esclarecer las posibles contribuciones del bilingüismo a la preservación de las funciones cognitivas. De este modo, se podría arrojar luz sobre la relación entre el bilingüismo y la $\mathrm{RC}, \mathrm{y}$, con ello, sobre los mecanismos de compensación cognitiva en su conjunto.

Referencias bibliográficas

Abutalebi, J., Canini, M., Della Rosa, P. A., Green, D. W., and Weekes, B. S. (2015). The neuroprotective effects of bilingualism upon the inferior parietal lobule: a structural neuroimaging study in aging Chinese bilinguals. Journal of Neurolinguistics, 33, 3-13.

Abutalebi, J., Canini, M., Della Rosa, P. A., Sheung, L. P., Green, D. W., and Weekes, B. S. (2014). Bilingualism protects anterior temporal lobe integrity in aging. Neurobiology of Aging, 35(9), 2126-2133.

Adi-Japha, E., Berberich-Artzi, J., and Libnawi, A. (2010). Cognitive flexibility in drawings of bilingual children. Child Development, 81(5), 1356-1366.

Albert, M. S., DeKosky, S. T., Dickson, D., Dubois, B., Feldman, H. H., Fox, N. C., y otros (2011). The diagnosis of mild cognitive impairment due to Alzheimer's disease: Recommendations from the National Institute on Aging-Alzheimer's Association workgroups on diagnostic guidelines for Alzheimer's disease. Alzheimer's y Dementia, 7, 270-279.

Alladi, S., Bak, T. H., Duggirala, V., Surampudi, B., Shailaja, M., Shukla, A. K., y otros (2013). Bilingualism delays age at onset of dementia, independent of education and immigration status. Neurology, 81(22), 1938-1944.

Amieva, H., Jacqmin-Gadda, H., Orgogozo, J. M., Le Carret, N., Helmer, C., Letenneur, L., y otros (2005). The 9 year cognitive decline before dementia of the Alzheimer type: a prospective population-based study. Brain, 128(5), 1093-1101. 
Ardal, S., Donald, M. W., Meuter, R., Muldrew, S., and Luce, M. (1990). Brain responses to semantic incongruity in bilinguals. Brain and language, 39(2), 187-205.

Ardila, Alfredo (2012). Ventas y desventajas del bilingüismo. Forma y función 25(2), 99-114.

Bajo, M.T., Padilla, F., and Padilla, P. (2000). Comprehension processes in simultaneous interpreting. Benjamins Translation Library, 39, 127-142.

Bak, T. H., Nissan, J. J., Allerhand, M. M., and Deary, I. (2014). Does bilingualism influence cognitive aging? Annals of Neurology, 75, 959-963.

Bennett, D. A., Schneider, J. A., Tang, Y., Arnold, S. E., and Wilson, R. S. (2006). The effect of social networks on the relation between Alzheimer's disease pathology and level of cognitive function in old people: a longitudinal cohort study. The Lancet Neurology, $5(5), 406-412$.

Bethel, J. W., and Schenker, M. B. (2005). Acculturation and smoking patterns among Hispanics: a review. American Journal of Preventive Medicine, 29(2), 143-148.

Bhugra, D., and Becker, M. A. (2005). Migration, cultural bereavement and cultural identity. World Psychiatry, 4(1), 18.

Bialystok, E., Craik F. I. M., Klein., R, and Viswanathan, M. (2004). Bilingualism, aging, and cognitive control: Evidence from the Simon task. Psychology and Aging, 19(2), 290-303.

Bialystok, E., Craik, F., and Freedman, M. (2007). Bilingualism as a protection against the onset of symptoms of dementia. Neuropsychologia, 45, 459-464.

Bialystok, E., Craik, F. I. M., and Luk, G. (2008). Cognitive control and lexical access in younger and older bilinguals. Journal of Experimental Psychology: Learning, Memory, and Cognition, 34, 859-873.

Bialystok, E., Craik, F. I. M., Green, D. W., and Gollan, T. H. (2009). Bilingual minds. Psychological Science in the Public Interest, 10, 89-129.

Bialystok, E. (2011). Coordination of executive functions in monolingual and bilingual children. Journal of Experimental Child Psychology, 461-468.

Bialystok, E., Craik, F. I., and Luk, G. (2012). Bilingualism: consequences for mind and brain. Trends in Cognitive Sciences, 16(4), 240-250. 
Bialystok, E., Craik, F. I. M., Binns, M. A., Ossher, L., and Freedman, M. (2014). Effects of bilingualism on the age of onset and progression of MCI and AD: Evidence from executive function tests. Neuropsychology, 28, 290-304.

Bloomfield, L. (1935). Language. Londres: Allen y Unwin.

Bonifacci, P., Giombini, L., Bellocchi, S., and Contento, S. (2010). Speed of processing, anticipation, inhibition and working memory in bilinguals. Developmental Science, 14(2), 256-269.

Brockmole, J.R, and Logie, R.H. (2013). Age-related change in visual working memory: a study of 55,753 participants aged 8-75. Frontiers in psychology, 4: 12.

Brockmole, J.R, Parra, M.A, Della Sala, S., and Logie, R.H. (2008). Do binding deficits account for age-related decline in visual working memory? Psychonomic Bulletin $y$ Review, 15(3): 543-7.

Brown, L.A., and Brockmole, J.R. (2010). The role of attention in binding visual features in working memory: evidence from cognitive ageing. Quarterly Journal of Experimental Psychology, 63(10): 2067-79.

Bowles, R. P., and Salthouse, T. A. (2003). Assessing the age-related effects of proactive interference on working memory tasks using the Rasch model. Psychology and Aging, $18,608-615$.

Buckner, R. L. (2004). Memory and executive function in aging and AD: multiple factors that cause decline and reserve factors that compensate. Neuron, 44(1), 195-208.

Bulik, C. M., Sullivan, P. F., Tozzi, F., Furberg, H., Lichtenstein, P., and Pedersen, N. L. (2006). Prevalence, heritability, and prospective risk factors for anorexia nervosa. Archives of General Psychiatry, 63(3), 305-312.

Burvill, P. W. (1993). A critique of current criteria for early dementia in epidemiological studies. International Journal of Geriatric Psychiatry, 8, 553-559.

Cabeza, R., and Dennis, N.A. (2013). "Frontal lobes and aging: Deterioration and compensation" in Principles of Frontal Lobe Function, ed., D.T., Stuss, and R.T., Knight. (New York: Oxford University Press), 628-652. 
Caetano, R., Ramisetty-Mikler, S., Rodriguez, L.A. (2008). The Hispanic Americans Baseline Alcohol Survey (HABLAS): rates and predictors of alcohol abuse and dependence across Hispanic national groups. Journal of Studies on Alcohol and Drugs, 69, 441-448.

Calvo, N., García, A. M., Manoiloff, L., e Ibáñez, A. (2015). Bilingualism and Cognitive Reserve: A Critical Overview and a Plea for Methodological Innovations. Frontiers in Aging Neuroscience, 7, 249, 1-17, http://doi.org/10.3389/fnagi.2015.00249, https://www.ncbi.nlm.nih.gov/pmc/articles/PMC4709424/.

Calvo, N., Ibáñez, A. y García, A. M. (2016). The impact of bilingualism on working memory: A null effect on the whole may not be so on the parts. Frontiers in Psychology: Language Sciences 7, 265.

Carlson, S. M., and Meltzoff, A. N. (2008). Bilingual experience and executive functioning in young children. Developmental science, 11(2), 282-298.

Clare, L., Whitaker, C. J., Craik, F. I., Bialystok, E., Martyr, A., Martin - Forbes, P., y otros (2014). Bilingualism, executive control, and age at diagnosis among people with early-stage Alzheimer's disease in Wales. Journal of Neuropsychology. 2016 Sep;10(2):163-85. doi: 10.1111/jnp.12061. Epub 2014 Nov 25.

Clark, C. M., Sheppard, L., Fillenbaum, G. G., Galasko, D., Morris, J. C., Koss, E., y otros (1999). Variability in annual Mini-Mental State Examination score in patients with probable Alzheimer disease: a clinical perspective of data from the Consortium to Establish a Registry for Alzheimer's Disease. Archives of Neurology, 56(7), 857-862.

Craft, G. E., Chen, A., and Nairn, A. C. (2013). Recent advances in quantitative neuroproteomics. Methods, 61(3), 186-218.

Craik, F. I. M., Bialystok, E., and Freedman, M. (2010). Delaying the onset of Alzheimer disease: Bilingualism as a form of cognitive reserve. Neurology, 75, 1726-1729.

Crane, P. K., Gruhl, J. C., Erosheva, E. A., Gibbons, L. E., McCurry, S. M., Rhoads, K., y otros (2010). Use of spoken and written Japanese did not protect Japanese-American men from cognitive decline in late life. Journal of Gerontology, Series B: Psychological Sciences and Social Sciences, 65, 654-666. 
Crocco, E., Curiel, R. E., Acevedo, A., Czaja, S. J., and Loewenstein, D. A. (2014). An evaluation of deficits in semantic cueing and proactive and retroactive interference as early features of Alzheimer's disease. The American Journal of Geriatric Psychiatry, 22(9), 889-897.

Codó, E. (2008). "Interviews and questionnaires" in The Blackwell Guide to Research Methods in Bilingualism and Multilingualism, eds. L. Wei and M. G. Moyer (Oxford: Blackwell), 158-176.

Cooper, P. S., Wong, A. S., Fulham, W. R., Thienel, R., Mansfield, E., Michie, P. T., and Karayanidis, F. (2015). Theta frontoparietal connectivity associated with proactive and reactive cognitive control processes. NeuroImage, 108, 354-363.

Costa, A., Hernández, M., and Sebastián-Gallés, N. (2008). Bilingualism aids conflict resolution: Evidence from the ANT task. Cognition, 106(1), 59-86.

Dalrymple-Alford, J. C., MacAskill, M. R., Nakas, C. T., Livingston, L., Graham, C., Crucian, G. P. and Anderson, T. J. (2010). The MoCA: well-suited screen for cognitive impairment in Parkinson disease. Neurology, 75(19), 1717-1725.

Davenport, M. H., Hogan, D. B., Eskes, G. A., Longman, R. S., and Poulin, M. J. (2012). Cerebrovascular reserve: the link between fitness and cognitive function? Exercise and Sport Sciences Reviews, 40(3), 153-158.

Díaz-Urueta, U., Buiza-Bueno, C., y Yanguas-Lezaun, J. (2010). Reserva cognitiva: evidencias, limitaciones y líneas de investigación futura. Revista Española de Geriatría y Gerontología, 45(3), 150-155.

De Bot, K., and Jaensch, C. (2015). What is special about L3 processing? Bilingualism: Language and Cognition, 1-15.

Duñabeitia, J. A., and Carreiras, M. (2015). The bilingual advantage: Acta est fabula? Cortex, 73 .

Dunn, L. M., and Dunn, L. M. (1997). Examiner's manual for the PPVT-III Peabody Picture Vocabulary Test: Form IIIA and Form IIIB. AGS.

Ebert, P. L., and Anderson, N. D. (2009). Proactive and retroactive interference in young adults, healthy older adults, and older adults with amnestic mild cognitive impairment. Journal of the International Neuropsychological Society, 15(01), 83-93. 
Engel de Abreu, P. M. (2011). Working memory in multilingual children: Is there a bilingual effect? Memory, 19(5), 529-537.

Epstein, J. A., Dusenbury, L., Botvin, G., and Diaz, T. (1996). Language use and initiation of alcohol use among New York City Hispanic adolescents. Journal of Child y Adolescent Substance Abuse, 5(2), 63-79.

Erickson, K. I., Voss, M. W., Prakash, R. S., Basak, C., Szabo, A., Chaddock, L., y otros (2011). Exercise training increases size of hippocampus and improves memory. Proceedings of the National Academy of Sciences, 108(7), 3017-3022.

Fairburn, C. G., and Beglin, S. J. (1994). Assessment of eating disorders: Interview or self - report questionnaire? International Journal of Eating Disorders, 16(4), 363-370.

Feher, E. P., Mahurin, R. K., Doody, R. S., Cooke, N., Sims, J., and Pirozzolo, F. J. (1992). Establishing the limits of the Mini-Mental State: Examination of 'subtests'. Archives of Neurology, 49(1), 87-92.

Feng, X. (2009). Working memory and bilingualism: An investigation of executive control and processing speed. ProQuest.

Folstein, M. F., Folstein, S. E., and McHugh, P. R. (1975). Mini-mental state. A practical method for grading the cognitive state of patients for the clinician. Journal of Psychiatric Research, 12, 189-198.

Fuller-Thomson, E., and Kuh, D. (2014). The healthy migrant effect may confound the link between bilingualism and delayed onset of Alzheimer's disease. Cortex, 52, 128-130.

García, A. M. (2014b). Neurocognitive determinants of performance variability among world-language users. Journal of World Languages, 1(1), 60-77.

García, A. M., Manoiloff, L. y Wagner, M. (2016). Concepciones del bilingüismo y evaluación de la competencia bilingüe. En Adolfo M. García y Sonia Suárez Cepeda (eds.), Mente bilingüe: abordajes psicolingüísticos y cognitivistas (pp. 17-49). Córdoba: Comunicarte.

García-Pentón, L., Fernández García, Y., Costello, B., Duñabeitia, J. A., and Carreiras, M. (2015). The neuroanatomy of bilingualism: How to turn a hazy view into the full picture. Language, Cognition and Neuroscience, 1-25. doi:10.1080/ 23273798.2015 .1068944$. 
Gelfo, F., De Bartolo, P., Giovine, A., Petrosini, L., and Leggio, M. G. (2009). Layer and regional effects of environmental enrichment on the pyramidal neuron morphology of the rat. Neurobiology of Learning and Memory, 91(4), 353-365.

Geller, G., and Thomas, C. D. (1999). A review of eating disorders in immigrant women: Possible evidence for a culture-change model. Eating Disorders, 7(4), 279-297.

Ghidoni, R., Paterlini, A., Albertini, V., Binetti, G., and Benussi, L. (2012). Losing protein in the brain: the case of progranulin. Brain research, 1476: 172-82.

Gómez-Ruiz, M.I. (2010). “Bilingüismo y cerebro: Mito y realidad”. Neurología 25(7):443452.

Grady, C. L., Luk, G., Craik, F. I., and Bialystok, E. (2015). Brain network activity in monolingual and bilingual older adults. Neuropsychologia, 66, 170-181.

Green, M. and Rogers, P. (1998). Impairments in working memory associated with spontaneous dieting behaviour. Psychological Medicine, 28, 1063-1070.

Gleichgerrcht, E., Roca, M., Manes, F., and Torralva, T. (2011). Comparing the clinical usefulness of the Institute of Cognitive Neurology (INECO) Frontal Screening (IFS) and the Frontal Assessment Battery (FAB) in frontotemporal dementia. Journal of Clinical and Experimental Neuropsychology, 33(9), 997-1004.

Gollan, T. H., Salmon, D. P., Montoya, R. I., and Galasko, D. R. (2011). Degree of bilingualism predicts age of diagnosis of Alzheimer's disease in low-education but not in highly educated Hispanics. Neuropsychologia, 49(14), 3826-3830.

Gold, B. T., Kim, C., Johnson, N. F., Kryscio, R. J., and Smith, C. D. (2013). Lifelong Bilingualism Maintains Neural Efficiency for Cognitive Control in Aging. The Journal of Neuroscience: The Official Journal of the Society for Neuroscience, 33(2), 387-396.

Grober, E., Hall, C. B., Lipton, R. B., Zonderman, A. B., Resnick, S. M., and Kawas, C. (2008). Memory impairment, executive dysfunction, and intellectual decline in preclinical Alzheimer's disease. Journal of the International Neuropsychological Society, 14(2), 266-278.

Grosjean, F. (1994). "Individual bilingualism" in The Encyclopaedia of Language and Linguistics, ed. R. E. Asher (Oxford: Pergamon Press), 1656-1660. 
Henn, C., Loffelholz, K., and Klein, J. (1998) Stimulatory and inhibitory effects of ethanol on hippocampal acetylcholine release. Naunyn Schmiedebergs Archive Pharmacology, $357,640-647$.

Hindle, J. V., Martin-Forbes, P. A., Bastable, A. J. M., Pye, K. L., Martyr, A., Whitaker, C., y otros (2015). Cognitive Reserve in Parkinson's Disease: The effects of Welsh-English bilingualism on executive function. Parkinson's Disease, 2015, 943572.

Hogervorst, E., Huppert, F., Matthews, F. E., and Brayne, C. (2008). Thyroid function and cognitive decline in the MRC Cognitive Function and Ageing Study. Psychoneuroendocrinology, 33(7), 1013-1022.

Hulstijn, J. H. (2010). "Measuring second language proficiency", in Experimental Methods in Language Acquisition Research, eds. E. Blom and S. Unsworth (Amsterdam: John Benjamins), 185-199.

Hulstijn, J. H. (2012). The construct of language proficiency in the study of bilingualism from a cognitive perspective. Bilingualism: Language and Cognition, 15(2), 422-433.

Hoops, S., Nazem, S., Siderowf, A. D., Duda, J. E., Xie, S. X., Stern, M. B., and Weintraub, D. (2009). Validity of the MoCA and MMSE in the detection of MCI and dementia in Parkinson disease. Neurology, 73(21), 1738-1745.

Kadam, P., and Bhalerao, S. (2010). Sample size calculation. International Journal of Ayurveda Research, 1(1), 55-57.

Karama, S., Ducharme, S., Corley, J., Chouinard-Decorte, F., Starr, J. M., Wardlaw, J. M. y otros (2015). Cigarette smoking and thinning of the brain's cortex. Molecular Psychiatry.

Karch, C.M., Cruchaga, C., Goate, A.M. (2014). Alzheimer's Disease Genetics: From the Bench to the Clinic. Neuron, 83(1): 11-26.

Kave, G., Eyal, N., Shorek, A., and Cohen-Mansfield, J. (2008). Multilingualism and cognitive state in the oldest old. Psychology and Aging, 23, 70-78.

Kim, K. H., Relkin, N. R., Lee, K. M., and Hirsch, J. (1997). Distinct cortical areas associated with native and second languages. Nature, 388(6638), 171-174.

Kim, S. Y., and Caine, E. D. (2014). Utility and limits of the mini mental state examination in evaluating consent capacity in Alzheimer's disease. Psychiatric Services. 
Kovacs, G.G., Botond, G., and Budka, H. (2010). Protein coding of neurodegenerative dementias: the neuropathological basis of biomarker diagnostics. Acta Neuropathologica, 119(4): 389-408.

Kowoll, M. E., Degen, C., Gladis, S., and Schröder, J. (2015). Neuropsychological profiles and verbal abilities in lifelong bilinguals with mild cognitive impairment and Alzheimer's disease. Journal of Alzheimer's disease, 45(4), 1257-1268.

Kousaie, S., and Phillips, N. A. (2012). Ageing and bilingualism: Absence of a "bilingual advantage" in Stroop interference in a nonimmigrant sample. The Quarterly Journal of Experimental Psychology, 65, 356-369.

Lawton, D. M., Gasquoine, P. G., and Weimer, A. A. (2015). Age of dementia diagnosis in community dwelling bilingual and monolingual Hispanic Americans. Cortex, $66,141-145$.

Little, T. D. (2013). The Oxford Handbook of Quantitative Methods in Psychology, volume 1. Oxford: Oxford University Press.

Lustig, C., May, C. P., and Hasher, L. (2001). Working memory span and the role of proactive interference. Journal of Experimental Psychology General, 130, 199-207.

Lustig, C., and Hasher, L. (2002). Working memory span: The effect of prior learning. American Journal of Psychology, 115(1), 89-101.

Mackey, W. F. (1968). "The description of bilingualism" in Readings in the Sociology of Language, eds J. Fishma (La Haya: Mouton), pp. 554-584.

Marian, V., Blumenfeld, H. K., Mizrahi, E., Kania, U., and Cordes, A. K. (2013). Multilingual Stroop performance: Effects of trilingualism and proficiency on inhibitory control. International Journal of Multilingualism, 10(1), 82-104.

Mattis, S. (1998). Dementia Rating Scale: Professional Manual. Florida: Odessa.

McKhann, G., Drachman, D., Folstein, M., Katzman, R., Price, D., and Stadlan, E. M., (1984). Clinical-diagnosis of Alzheimer's disease: Report of the NINCDS-ADRDA work group under the auspices of Department of Health and Human Services task-force on Alzheimer's disease. Neurology, 34, 939-944.

Meara, P. (1994). LLEX: Lingua Vocabulary Test v. 1.4. Swansea: Center for Applied Linguistics, University of Wales. 
Michon, M. (2016). Consecuencias del bilingüismo sobre la cognición. En Adolfo M. García y Sonia Suárez Cepeda (eds.), Mente bilingüe: abordajes psicolingüísticos y cognitivistas (pp. 145-142). Córdoba: Comunicarte.

Mohs, R. C., Rosen, W. G., and Davis K. L. (1983). The Alzheimer's Disease Assessment Scale: an instrument for assessing treatment efficacy. Psychopharmacology Bulletin, 19, 448-450.

Mohs, R. C., Cohen L. (1988). Alzheimer's Disease Assessment Scale (ADAS). Psychopharmacology Bulletin, 24, 627-628.

Mohs, R.C. (1994). Administration and Scoring Manual for the Alzheimer's Disease Assessment Scale, revised Edition. New York: The Mount Sinai School of Medicine.

Morales, J., Calvo, A., and Bialystok, E. (2013a). Working memory development in monolingual and bilingual children. Journal of Experimental Child Psychology, $114,187-202$.

Morales, J., Gómez-Ariza, C., and Bajo T, M. (2013b). Dual mechanisms of cognitive control in bilinguals and monolinguals. Journal of Cognitive Psychology, 25(5), 531-546. doi:10.1080/20445911.2013.807812

Namazi, M., and Thordardottir, E.T. (2010). A working memory, not bilingual advantage, in controlled attention. International Journal of Bilingual Education and Bilingualism, 13(5), 597-616.

Nasreddine, Z. S., Phillips, N. A., Bedirian, V., Charbonneau, S., Whitehead, V., Collin, I., and Chertkow, H. (2005). The Montreal Cognitive Assessment, MoCA: a brief screening tool for mild cognitive impairment. Journal of the American Geriatrics Society, 53(4), 695-699.

Neville, H. J., Mills, D. L., and Lawson, D. S. (1992). Fractionating language: Different neural subsystems with different sensitive periods. Cerebral Cortex, 2(3), 244-258.

Neville, H. J., Coffey, S. A., Lawson, D. S., Fischer, A., Emmorey, K., and Bellugi, U. (1997). Neural systems mediating American Sign Language: Effects of sensory experience and age of acquisition. Brain and language, 57(3), 285-308. 
Olsen, R. K., Pangelinan, M. M., Bogulski, C., Chakravarty, M. M., Luk, G., Grady, C. L., and Bialystok, E. (2015). The effect of lifelong bilingualism on regional grey and white matter volume. Brain Research, 1612, 128-139.

Ossher, L., Bialystok, E., Craik, F. I. M., Murphy, K. J., and Troyer, A. K. (2013). The effect of bilingualism on amnestic mild cognitive impairment. Journal of Gerontology, Series B: Psychological Sciences and Social Sciences, 68, 8-12.

Paradis, M. (2009). Declarative and Procedural Determinants of Second Languages. Amsterdam: John Benjamins.

Paradis, M. (2011). Principles underlying the Bilingual Aphasia Test (BAT) and its uses. Clinical Linguistics y Phonetics, 25(6-7), 427-443.

Paradis, M. and Lecours, A. R. (1979). L'aphasie chez les bilingues et les polyglotes. In L'aphasie, eds. A.R. Lecours and F. Lhermitte, (Paris: Flammarion), pp. 605-616.

Parra, M. A., Abrahams, S., Fabi, K., Logie, R., Luzzi, S., and Della Sala, S. (2009). Shortterm memory binding deficits in Alzheimer's disease. Brain, awp036.

Parra, M. A., Abrahams, S., Logie, R. H., Mendez, L. G., Lopera, F., and Della Sala, S. (2010). Visual short-term memory binding deficits in familial Alzheimer's disease. Brain: a Journal of Neurology, 133(9), 2702-1273.

Perani, D., Dehaene, S., Grassi, F., Cohen, L., Cappa, S. F., Dupoux, E., y otros (1996). Brain processing of native and foreign languages. Neuroreport, 7(15-17), 2439-2444.

Petersen, R. C. (2004). Mild cognitive impairment as a diagnostic entity. Journal of Internal Medicine, 256, 183-194.

Prior, A., and MacWhinney, B. (2010). A bilingual advantage in task switching. Bilingualism: Language and Cognition, 13(02), 253-262.

Robins, L.N., Wing, J., Wittchen, H.U., Helzer, J.E., Babor, T.F., Burke, J., y otros (1989). The Composite International Diagnostic Interview: An epidemiologic instrument suitable for use in conjunction with different diagnostic systems and in different cultures. Archives of General Psychiatry, 45, 1069-1077.

Roca, M., Torralva, T., Gleichgerrcht, E., Woolgar, A., Thompson, R., Duncan, J., and Manes, F. (2011). The role of area 10 (BA10) in human multitasking and in social cognition: a lesion study. Neuropsychologia, 49(13), 3525-3531. 
Rodriguez - Fornells, A., De Diego Balaguer, R., and Münte, T. F. (2006). Executive control in bilingual language processing. Language Learning, 56(1), 133-190.

Salvatierra, J. L., and Rosselli, M. (2010). The effect of bilingualism and age on inhibitory control. International Journal of Bilingualism.

Sanders, A. E., Hall, C. B., Katz, M. J., and Lipton, R. B. (2012). Non-native language use and risk of incident dementia in the elderly. Journal of Alzheimer's Disease, 29, 99-108.

Sattler, C., Toro, P., Schönknecht, P., and Schröder, J. (2012). Cognitive activity, education and socioeconomic status as preventive factors for mild cognitive impairment and Alzheimer's disease. Psychiatry Research, 196(1), 90-95.

Scarmeas, N., and Stern, Y. (2004). Cognitive reserve: implications for diagnosis and prevention of Alzheimer's disease. Current Neurology and Neuroscience Reports, 4(5), 374-380.

Schweizer, T.A., Ware, J., Fischer, C.E., Craik, F.I., and Bialystok, E. (2012). Bilingualism as a contributor to cognitive reserve: evidence from brain atrophy in Alzheimer's disease. Cortex, 48, 991-996.

Sheline, Y.I., and Raichle, M.E. (2013). Resting state functional connectivity in preclinical Alzheimer's disease. Biological Psychiatry, 74(5), 340-347.

Shiroky, J.S., Schipper, H.M., Bergman, H., Chertkow, H. (2007). Can you have dementia with an MMSE score of 30? American Journal of Alzheimer's Disease and Other Dementias, 22(5), 406-415.

Staudinger, U. M., Marsiske, M., and Baltes, P. B. (1993). Resilience and levels of reserve capacity in later adulthood: Perspectives from life-span theory. Development and Psychopathology, 5(04), 541-566.

Stevenson, J. R., Schroeder, J. P., Nixon, K., Besheer, J., Crews, F. T., and Hodge, C. W. (2009). Abstinence following alcohol drinking produces depression-like behavior and reduced hippocampal neurogenesis in mice. Neuropsychopharmacology, 34(5), 1209-1222. 
Stein, M., Federspiel, A., Koenig, T., Wirth, M., Strik, W., Wiest, R., Brandeis, D., and Dierks, T. (2012). Structural plasticity in the language system related to increased second language proficiency. Cortex, 48(4), 458-465.

Stern, Y. (2009). Cognitive reserve. Neuropsychologia, 47(10), 2015-2028.

Stern, Y. (2012). Cognitive reserve in ageing and Alzheimer's disease. Lancet Neurology, 11(11), 1006-1012.

Sullivan, G. M., and Feinn, R. (2012). Using effect size-or why the P value is not enough. Journal of Graduate Medical Education, 4(3), 279-282.

Szaflarski, M., Cubbins, L. A., and Ying, J. (2011). Epidemiology of Alcohol Abuse Among US Immigrant Populations. Journal of Immigrant and Minority Health / Center for Minority Public Health, 13(4), 647-658.

Tao, L., Marzecová, A., Taft, M., Asanowicz, D., and Wodniecka, Z. (2011). The efficiency of attentional networks in early and late bilinguals: the role of age of acquisition. Frontiers in Psychology, 2.

Tang-Wai, D. F., Knopman, D. S., Geda, Y. E., Edland, S. D., Smith, G. E., Ivnik, R. J., y otros (2003). Comparison of the short test of mental status and the mini-mental state examination in mild cognitive impairment. Archives of Neurology, 60(12), 1777-1781.

Tractenberg, R. E., Singer, C. M., and Kaye, J. A. (2006). Characterizing sleep problems in persons with Alzheimer's disease and normal elderly. Journal of Sleep Research, 15(1), 97-103.

Torralva, T., Roca, M., Gleichgerrcht, E., Lopez, P., and Manes, F. (2009a). INECO Frontal Screening (IFS): a brief, sensitive, and specific tool to assess executive functions in dementia. Journal of the International Neuropsychological Society, 15(5), 777-786.

Torralva, T., Roca, M., Gleichgerrcht, E., Bekinschtein, T., and Manes, F. (2009b). A neuropsychological battery to detect specific executive and social cognitive impairments in early frontotemporal dementia. Brain, 132(5), 1299-1309.

Ullman, M. T. (2001). The neural basis of lexicon and grammar in first and second language: The declarative/procedural model. Bilingualism: Language and Cognition, 4(1), 105-122. 
Unger, J. B., Cruz, T. B., Rohrbach, L. A., Ribisl, K. M., Baezconde-Garbanti, L., Chen, X., y otros (2000). English language use as a risk factor for smoking initiation among Hispanic and Asian American adolescents: evidence for mediation by tobacco-related beliefs and social norms. Health Psychology, 19(5), 403.

Valdés, G. and Figueroa, R.A. (1994). Bilingualism and Testing: A Special Case of Bias. Norwood. New Jersey: Ablex Publishing.

Voss, U., and Tuin, I. (2008). Integration of immigrants into a new culture is related to poor sleep quality. Health and Quality of Life Outcomes, 6(1), 61.

Walker, M.P. and Stickgold, R. (2006). Sleep, memory and plasticity. Annual Review of Psychology, 10, 139-166.

Weber-Fox, C. M. and Neville, H. J. (1997). Maturational constraints on functional specializations for language processing: ERP and behavioral evidence in bilingual speakers. Journal of Cognitive Neuroscience, 8, 231-256.

Weinreich, U. (1953). Languages in Contact: Findings and Problems. La Haya: Mouton.

White, H., Boden-Albala, B., Wang, C., Elkind, M.S., Rundek ,T., Wright, C.B., y otros (2005). Ischemic stroke subtype incidence among whites, blacks, and Hispanics: The Northern Manhattan Study. Circulation, 111, 1327 - 1331.

Woumans, E., Santens, P., Sieben, A., Versijpt, J., Stevens, M., and Duyck, W. (2015). Bilingualism delays clinical manifestation of Alzheimer's disease. Bilingualism: Language and Cognition, 18(03), 568-574.

Wind, A. W., Schellevis, F. G., Van Staveren, G. E., Scholten, R. J., Jonker, C., and Van Eijk, J. T. (1997). Limitations of the Mini - Mental State Examination in diagnosing dementia in general practice. International Journal of Geriatric Psychiatry, 12(1), 101-108.

Zahodne, L. B., Schofield, P. W., Farrell, M. T., Stern, Y., and Manly, J. J. (2014). Bilingualism does not alter cognitive decline or dementia risk among Spanish speaking immigrants. Neuropsychology, 28(2), 238-246.

Zahuranec, D. B., Brown, D. L., Lisabeth, L. D., Gonzales, N. R., Longwell, P. J., Eden, S. V., y otros (2006). Differences in intracerebral hemorrhage between Mexican Americans and non-Hispanic whites. Neurology, 66(1), 30-34. 
Zammit, G., Weiner, J., Damato, N., Sillup, G., McMillan, C. (1999). Quality of life in people with insomnia. Sleep, 22(2), 379-385.

Zhang, J., Goodlett, D. R., and Montine, T. J. (2005). Proteomic biomarker discovery in cerebrospinal fluid for neurodegenerative diseases. Journal of Alzheimer's Disease, 8(4), 377-386.

Agradecimientos

Este trabajo es una traducción revisada y aumentada de Calvo y otros (2015).

Información sobre los autores

Noelia Calvo,, ${ }^{1,2}$ Laura Manoiloff, ${ }^{2}$ Edinson Muñoz, ${ }^{3}$ Marcela Contreras, ${ }^{3}$ Agustín Ibáñez, ${ }^{4,5,6,7,8}$ Adolfo M. García, 4,5,9

${ }^{1}$ U. Nacional de San Juan, ${ }^{2}$ U. Nacional de Córdoba, ${ }^{3}$ Universidad de Santiago de Chile, ${ }^{4}$ Universidad Favaloro, ${ }^{5}$ CONICET, ${ }^{6}$ U. Autónoma del Caribe, ${ }^{7}$ U. Adolfo Ibáñez, ${ }^{8}$ Australian Research Council, ${ }^{9}$ Universidad Nacional de Cuyo 
Anexo

Tabla 1. Resumen de estudios retrospectivos sobre la relación entre bilingüismo y reserva cognitiva en poblaciones con demencia

\begin{tabular}{|c|c|c|c|c|}
\hline Muestra & $\begin{array}{c}\text { Evaluación } \\
\text { de bilingüismo y } \\
\text { competencia } \\
\text { lingüística }\end{array}$ & $\begin{array}{c}\text { Control } \\
\text { de variables } \\
\text { que afectan la } \mathrm{RC}\end{array}$ & $\begin{array}{l}\text { Evaluación } \\
\text { de demencia } \\
\text { o cognición }\end{array}$ & Resultados \\
\hline \multicolumn{5}{|c|}{ Bialystok y otros (2007) } \\
\hline $\begin{array}{l}91 \text { ML con EA (48 m) } \\
\text { Edad de aparición: } 71,4 \\
\text { Edad en la primera con: } 75,4 \\
\text { Años de educación: } 12,4 \\
\text { MMSE en la primera con: } 21,3 \\
\text { Estado ocupacional*: } 3,3 \\
93 \text { BL con EA ( } 55 \mathrm{~m}) \\
\text { (todos IN: inglés y cualquier } \\
\text { otra leng) } \\
\text { Edad de aparición: } 75,5 \\
\text { Edad en la primera con: } 78,6 \\
\text { Años de educación: } 10,8 \\
\text { MMSE en la primera con: } 20,1 \\
\text { Estado ocupacional } *: 3\end{array}$ & $\begin{array}{l}\text { Evaluación subjetiva } \\
\text { de leng; fluidez en } \\
\text { Ing; lugar de } \\
\text { nacimiento; fecha de } \\
\text { nacimiento; año de } \\
\text { INM }\end{array}$ & $\begin{array}{l}\text { Años de educación y } \\
\text { estado ocupacional. } \\
\text { Algunos pacientes } \\
\text { padecían de ECV, } \\
\text { depresión, psicosis, } \\
\text { meningioma o apnea } \\
\text { de sueño. }\end{array}$ & $\begin{array}{l}\text { MMSE; } \\
\text { TC; } \\
\text { SPECT }\end{array}$ & $\begin{array}{l}\text { Mayor RC en BL } \\
\text { Los BL tenían al menos } \\
4 \text { años más a la edad de } \\
\text { aparición }\end{array}$ \\
\hline \multicolumn{5}{|c|}{ Cherktow y otros (2010) } \\
\hline $\begin{array}{l}379 \text { ML con EA }(240 \mathrm{~m}) \\
\text { (23I: } 66 \text { hablaban Fra, } 290 \\
\text { hablaban Ing) } \\
\text { Edad al diagnóstico: } 76,7 \\
\text { Años de educación: } 10,9 \\
\text { Puntaje en MMSE: } 23,1 \\
\text { 253 PL con EA (131 m) } \\
\text { (135 IN, } 19 \text { NIN con Ing como } \\
\text { L1) } \\
\text { Edad al diagnóstico: } 77,6 \\
\text { Años de educación: } 10,7 \\
\text { Puntaje en MMSE: } 22,9\end{array}$ & $\begin{array}{l}\text { Entrevistas con los } \\
\text { pacientes y los } \\
\text { cuidadores. } \\
\text { Estado de IN/ nativo } \\
\text { asumido. } \\
\text { EdA y edad de INM } \\
\text { no controlada. }\end{array}$ & $\begin{array}{l}\text { Igual que en } \\
\text { Bialystok } \\
\text { y otros (2007) }\end{array}$ & MMSE & $\begin{array}{l}\text { Mayor RC en algunos } \\
\text { BL } \\
3 \text { años de retraso en IN } \\
\text { BL y PL. No se } \\
\text { observó ningún } \\
\text { beneficio en NIN BL. } \\
\text { Se observó beneficios } \\
\text { en NIN cuya L1 era } \\
\text { Fra, pero no para } \\
\text { aquellos que su L1 era } \\
\text { Ing }\end{array}$ \\
\hline \multicolumn{5}{|c|}{ Craik y otros (2010) } \\
\hline $\begin{array}{l}\text { 109 ML con EA ( } 60 \mathrm{~m}) \\
\text { Edad a la aparición: } 72,6 \\
\text { Edad en la primera con: } 76,5 \\
\text { Duración: } 3,8 \\
\text { Años de educación: } 12,6 \\
\text { MMSE en la primera con: } 21,5 \\
\text { Estado ocupacional*: } 2,8 \\
\text { 102 BL con EA ( } 60 \mathrm{~m}) \\
\text { Edad a la aparición: } 77,7 \\
\text { Edad en la primera con: } 80,8 \\
\text { Duración: } 3,1 \\
\text { Años de educación: } 10,6 \\
\text { MMSE en la primera con: } 20,4 \\
\text { Estado ocupacional*: } 2,5\end{array}$ & $\begin{array}{l}\text { Igual que en } \\
\text { Bialystok } \\
\text { y otros (2007) }\end{array}$ & $\begin{array}{l}\text { Igual que en } \\
\text { Bialystok } \\
\text { y otros (2007) }\end{array}$ & MMSE & $\begin{array}{l}\text { Mayor RC en BL } \\
\text { A los BL se les había } \\
\text { diagnosticado } 4,3 \text { años } \\
\text { más tarde y habían } \\
\text { informado de la } \\
\text { aparición de los } \\
\text { síntomas } 5,1 \text { años más } \\
\text { tarde }\end{array}$ \\
\hline
\end{tabular}




\begin{tabular}{|c|c|c|c|c|}
\hline \multicolumn{5}{|c|}{ Crane y otros (2010) } \\
\hline $\begin{array}{l}\mathbf{2 5 2 0} 2^{\mathbf{a}} \text { generación BL J-EU } \\
\text { cf. (todos hombres) } \\
\mathbf{4 6 5} \text { ni hablaban ni leían Jap } \\
\text { Edad al examen HAAS 4: 76,1 } \\
\text { Educación: } 11,1 \\
\text { Puntaje CASI estándar en } \\
\text { examen HAAS 4: } 89 / 100 \\
\mathbf{1 4 9 5} \text { hablaban pero no leían } \\
\text { Jap } \\
\text { Edad al examen HAAS 4: 76,6 } \\
\text { Educación: } 10,8 \\
\text { Puntaje CASI estándar en } \\
\text { examen HAAS 4: } 87,4 / 100 \\
\text { 560 hablaban y leían Jap } \\
\text { Edad al examen HAAS 4: } 77,5 \\
\text { Educación: } 11,5 \\
\text { Puntaje CASI estándar en } \\
\text { examen HAAS 4: } 87\end{array}$ & $\begin{array}{l}\text { Dos preguntas sobre } \\
\text { habilidades orales y } \\
\text { escritas en Jap }\end{array}$ & $\begin{array}{l}\text { Educación, categoría } \\
\text { IA, análisis de } \\
\text { muestra sanguínea } \\
\text { para APOE, } \\
\text { perímetro cefálico, } \\
\text { tabaquismo (nunca, } \\
\text { pasado, actual) }\end{array}$ & $\begin{array}{l}\text { CASI: en HAAS } \\
\text { basal (edades 71- } \\
93 \text { ) y en tres } \\
\text { sondeos más: } \\
\text { Exámenes de } \\
\text { HAAS (edades74- } \\
95,77-98,79- \\
100) \text {; } \\
\text { DSM, TRI }\end{array}$ & $\begin{array}{l}\text { RC similar } \\
\text { No hubo evidencia que } \\
\text { apoyara la RC. } \\
\text { Las tasas de deterioro } \\
\text { cognitivo no se } \\
\text { relacionaron con el uso } \\
\text { de Jap hablado o } \\
\text { escrito }\end{array}$ \\
\hline \multicolumn{5}{|c|}{ Gollan y otros (2011) } \\
\hline $\begin{array}{l}\mathbf{2 2} \text { BL con alta educación con } \\
\text { EA } \\
\text { Edad de diagnóstico: } 75,1 \\
\text { Edad de aparición: } 72,1 \\
\text { Educación: } 14,6 \\
\text { MMSE: } 23,4 \\
\text { Índice+ bilingüe según TDB: } 64 \\
\text { Autoevaluados como bilingües: } \\
74 \\
\% \text { de uso diario de Ing: } 75,6 \\
\text { EdA de Ing: } 2,9 \\
\text { 22 BL con poca educación con } \\
\text { EA } \\
\text { Edad de diagnóstico: } 77,1 \\
\text { Edad de aparición: } 75 \\
\text { Educación: } 5,6 \\
\text { MMSE: } 24,2 \\
\text { Índice bilingüe según TDB: } \\
0,42 \\
\text { Índice de bilingüismo según } \\
\text { autoevaluación: } 0,52 \\
\text { \% de uso diario de Ing: } 21,1 \\
\text { EdA de Ing: } 19,9\end{array}$ & $\begin{array}{l}\text { Medición objetiva: } \\
\text { cálculo de puntajes } \\
\text { de índice bilingües a } \\
\text { través del TDB; } \\
\text { comparación de ese } \\
\text { puntaje con un índice } \\
\text { autoevaluado de } \\
\text { competencia bilingüe } \\
\text { hablada en cada leng }\end{array}$ & $\begin{array}{l}\text { Educación, grado de } \\
\text { bilingüismo }\end{array}$ & $\begin{array}{l}\text { MMSE, } \\
\text { DRS, } \\
\text { TDB }\end{array}$ & $\begin{array}{l}\text { Mayor RC en algunos } \\
\text { BL } \\
\text { Los mayores grados de } \\
\text { bilingüismo se } \\
\text { asociaron con una edad } \\
\text { de diagnóstico más } \\
\text { tardía, pero sólo en P } \\
\text { con nivel educativo } \\
\text { bajo (la mayoría } \\
\text { dominaba el Esp). } \\
\text { Sólo mediciones } \\
\text { objetivas, predicción de } \\
\text { la edad de aparición }\end{array}$ \\
\hline \multicolumn{5}{|c|}{ Sanders y otros (2012) } \\
\hline $\begin{array}{l}1389 \text { HNI }(845 \mathrm{~m}) \\
\text { Edad: } 78,3 \\
\text { Índice de incidencia de } \\
\text { demencia: } 2,87 \\
\text { Raza: }(n \text { blancos) } 928 \\
\text { Educación: } 13,3 \\
\text { Nivel de grado de lectura: } 12 \\
\text { Inmigrantes en EE. UU. : } 155 \\
\text { Casados: } 531 \\
\text { BIMC: } 2 \\
\text { TRLSF: } 29,9 \\
\text { GDS de } 15 \text { ítems: } 2 \\
\text { 390 Hn. NI }(236 \mathrm{~m})\end{array}$ & $\begin{array}{l}1 \text { pregunta en la } \\
\text { evaluación basal } \\
\text { sobre Ing como L1 } \\
\text { A los Hn. NI además } \\
\text { se les pidió definir su } \\
\text { L1, la edad a la que } \\
\text { aprendieron Ing y la } \\
\text { frecuencia de uso de } \\
\text { Ing. }\end{array}$ & $\begin{array}{l}\text { Información sobre } \\
\text { educación entregada } \\
\text { por los participantes; } \\
\text { hs médico entregado } \\
\text { por los pacientes } \\
\text { (diabetes, HTN, } \\
\text { derrame cerebral, } \\
\text { INM) }\end{array}$ & $\begin{array}{l}\text { Criterios DSM- } \\
\text { IV, BIMC, } \\
\text { inteligencia } \\
\text { premórbida, } \\
\text { atención, memoria } \\
\text { episódica, FE, } \\
\text { habilidad } \\
\text { visuoespacial y } \\
\text { leng; GDS de } 15 \\
\text { ítems }\end{array}$ & $\begin{array}{l}\text { RC similar } \\
\text { No se observa un } \\
\text { efecto protector contra } \\
\text { a EA en relación con el } \\
\text { estado de Hn. NI } \\
\text { (quizás de forma que } \\
\text { dependa de la } \\
\text { educación) }\end{array}$ \\
\hline
\end{tabular}




\begin{tabular}{|c|c|c|c|c|}
\hline $\begin{array}{l}\text { Edad: } 79,4 \\
\text { Índice de incidencia de } \\
\text { demencia: } 3,54 \\
\text { Raza: }(n \text { blancos) : } 317 \\
\text { Educación: } 12,5 \\
\text { Nivel de grado de lectura: } 12 \\
\text { Inmigrantes en EE. UU. : } 243 \\
\text { Casados: } 6 \\
\text { BIMC: } 2 \\
\text { TRLSF: } 30,1 \\
\text { GDS de } 15 \text { ítems: } 2\end{array}$ & & & & \\
\hline $\begin{array}{l}\text { 20 ML con EA }(14 \mathrm{~m}) \\
\text { Edad a la TC: } 77,2 \\
\text { Edad al diagnóstico: 77,3 } \\
\text { Educación: } 13,6 \\
\text { Estado ocupacional*: } 3,2 \\
\text { MMSE:23,2 } \\
\text { 20 BL con EA }(14 \mathrm{~m}) \\
\text { Edad a la TC: } 78,9 \\
\text { Edad al diagnóstico:78,9 } \\
\text { Educación: } 11,6 \\
\text { Estado ocupacional *: } 2,1 \\
\text { MMSE: } 22,1\end{array}$ & $\begin{array}{l}\text { En la mayoría de los } \\
\text { pacientes, el } \\
\text { bilingüismo se } \\
\text { confirmó a través del } \\
\text { cónyuge o el } \\
\text { cuidador }\end{array}$ & $\begin{array}{l}\text { Educación (los } \\
\text { grupos eran } \\
\text { similares), ocupación } \\
\text { (los grupos diferían) }\end{array}$ & $\begin{array}{l}\text { MMSE } \\
\text { CDR, Índice } \\
\text { Katzde AVD, } \\
\text { BNA y TDR }\end{array}$ & $\begin{array}{l}\text { Mayor RC en BL } \\
\text { Los BL mostraron } \\
\text { mayor atrofia cerebral } \\
\text { en la longitud radial del } \\
\text { asta temporal y en su } \\
\text { proporción }\end{array}$ \\
\hline \multicolumn{5}{|c|}{ Kousaie y Phillips (2012) } \\
\hline $\begin{array}{l}\text { 38 ML jóvenes saludables } \\
\text { Edad: } 22,5 \\
\text { Educación: } 15,1 \\
\text { MoCA: } 28,6 \\
\text { 35 ML jóvenes saludables } \\
\text { Edad: } 23,7 \\
\text { Educación: } 15,5 \\
\text { MoCA } 27,8 \\
\text { 25 ML mayores saludables } \\
\text { Edad: } 68,9 \\
\text { Educación: } 13,9 \\
\text { MoCA: } 26,8 \\
\text { 20 BL mayores saludables } \\
\text { Edad: } 71,9 \\
\text { Educación: } 15,9 \\
\text { MoCA: } 26,6\end{array}$ & $\begin{array}{l}\text { Tareas de decisión de } \\
\text { animacidad para } \\
\text { evaluar la } \\
\text { competencia relativa } \\
\text { en L1/ L2. } \\
\text { Autoevaluación de } \\
\text { comprensión } \\
\text { auditiva, lectura y } \\
\text { expresión oral en } \\
\text { cada leng en una } \\
\text { escala de } 15 \text { ítems }\end{array}$ & Educación & $\begin{array}{l}\text { MoCA, Test de } \\
\text { Stroop }\end{array}$ & 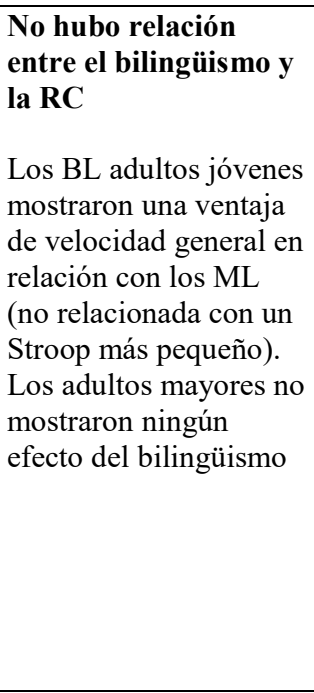 \\
\hline \multicolumn{5}{|c|}{ Alladi y otros (2013) } \\
\hline $\begin{array}{l}\text { 257 ML con EA (126 m) } \\
\text { Edad a la aparición: } 61,1 \\
\text { Edad a la pres: } 63,4 \\
\text { Años de educación: } 5,9 \\
\text { Ocupación: } 107 \\
\text { Alfabetización: } 177 \\
\text { En zonas urbanas: } 135 \\
\text { Duración: } 2,1 \\
\text { MMSE: } 16,7 \\
\text { 391 BL con EA (98 m) } \\
\text { Edad a la pres: } 68,1 \\
\text { Edad a la aparición: } 65,3 \\
\text { Años de educación: } 12,9 \\
\text { Ocupación: } 257 \\
\text { Alfabetización: } 373 \\
\text { En zonas urbanas: } 292 \\
\text { Duración: } 2,3 \\
\text { MMSE: } 18,9\end{array}$ & $\begin{array}{l}\text { Entrevista subjetiva a } \\
\text { un miembro de la } \\
\text { familia }\end{array}$ & $\begin{array}{l}\text { Sexo, alfabetización, } \\
\text { años de educación, } \\
\text { ocupación, vivienda } \\
\text { en área urbana o } \\
\text { rural, edad a la pres, } \\
\text { edad de aparición, } \\
\text { duración de la } \\
\text { enfermedad, puntaje } \\
\text { MMSE, ACE-R, } \\
\text { CDR, subtipo de } \\
\text { demencia (DFT, DV, } \\
\text { DCL, mixta), hs } \\
\text { familiar de demencia, } \\
\text { FRV, HTN, diabetes, } \\
\text { tabaquismo, } \\
\text { alcoholismo, EAC y } \\
\text { derrame cerebral }\end{array}$ & $\begin{array}{l}\text { MMSE } \\
\text { ACE-R } \\
\text { CDR (leve, } \\
\text { moderada, grave) }\end{array}$ & $\begin{array}{l}\text { Mayor RC in BL } \\
\text { Los BL eran mayores, } \\
\text { con más educación, } \\
\text { presentaban mejores } \\
\text { niveles de habilidades } \\
\text { en sus ocupaciones, etc. } \\
\text { Los BL presentaron } \\
\text { síntomas de demencia } \\
4,5 \text { años más tarde que } \\
\text { los ML }\end{array}$ \\
\hline
\end{tabular}




\begin{tabular}{|c|c|c|c|c|}
\hline \multicolumn{5}{|c|}{ Ossher y otros (2013) } \\
\hline $\begin{array}{l}49 \text { ML con DCL amnésico en } \\
\text { un solo dominio ( } 55 \% \text { m) } \\
\text { Edad: } 74,9 \\
\text { Duración de los síntomas: } 2,4 \\
\text { Educación: } 14,7 \\
\text { MMSE: } 27,7 \\
\text { 19 BL DCL amnésico en un } \\
\text { solo dominio ( } 32 \% \text { m) } \\
\text { Edad: } 79,4 \\
\text { Duración de los síntomas: } 2,8 \\
\text { Educación: } 14,5 \\
\text { MMSE: } 27,6 \\
\text { 22 ML con DCL amnésico en } \\
\text { múltiples dominios ( } 45 \% \text { m) } \\
\text { Edad: } 75,2 \\
\text { Duración de los síntomas:2,7 } \\
\text { Educación: } 14,9 \\
\text { MMSE: } 27,9 \\
\text { 21 BL DCL amnésico en } \\
\text { múltiples dominios ( } 43 \% \text { m) } \\
\text { Edad: } 72,6 \\
\text { Duración de los síntomas: } 2 \\
\text { Educación: } 15 \\
\text { MMSE: } 27,7\end{array}$ & $\begin{array}{l}\text { CST: L1 adquirida, } \\
\text { otras leng habladas, } \\
\text { frecuencia, etc. } \\
\text { Todos los pacientes } \\
\text { eran competentes en } \\
\text { Ing, pero los BL } \\
\text { hablaban una } \\
\text { variedad de otras } \\
\text { leng y no provenían } \\
\text { de ningún grupo } \\
\text { sociocultural } \\
\text { específico }\end{array}$ & $\begin{array}{l}\text { Antecedentes } \\
\text { sociales, } \\
\text { Educación }\end{array}$ & $\begin{array}{l}\text { MMSE } \\
2 \text { tests del D- } \\
\text { KEFS, } \\
\text { vocabulario, } \\
\text { HVTL (total y } \\
\text { recuperación con } \\
\text { retraso), amplitud } \\
\text { de dígitos, TDB, } \\
\text { ROCF, TMT, } \\
\text { Stroop, fluidez } \\
\text { verbal }\end{array}$ & $\begin{array}{l}\text { Mayor RC en BL } \\
\text { (solo en un tipo de } \\
\text { DCL amnésico) } \\
\text { Sólo aquellos con DCL } \\
\text { amnésico en un solo } \\
\text { dominio tuvieron una } \\
\text { edad de diagnóstico } \\
\text { más tardía en los BL } \\
\text { (79,4 años) que en los } \\
\text { ML ( } 74,9 \text { años) }\end{array}$ \\
\hline \multicolumn{5}{|c|}{ Zahodne y otros (2014) } \\
\hline $\begin{array}{l}\text { 637 ML inicialmente sin } \\
\text { demencia } \\
\text { ( } 72 \mathrm{~m}) \\
\text { Edad basal: } 75,66 \\
\text { Educación: } 5,05 \\
\text { Edad de INM: } 48,23 \\
\\
\text { 430 BL inicialmente sin } \\
\text { demencia }(64 \mathrm{~m}) \\
\text { Edad basal: } 74,78 \\
\text { Educación: } 8,3 \\
\text { Edad de INM:34,22 } \\
\end{array}$ & $\begin{array}{l}\text { Autoevaluación y } \\
\text { WRAT } 3\end{array}$ & Educación, INM & $\begin{array}{l}\text { DSM III, TRVS, } \\
\text { TDB de } 15 \text { ítems, } \\
\text { WAIS revisada, } \\
\text { CTT }\end{array}$ & $\begin{array}{l}\text { RC similar } \\
\text { Sin efecto protector del } \\
\text { bilingüismo }\end{array}$ \\
\hline \multicolumn{5}{|c|}{ Clare y otros (2014) } \\
\hline $\begin{array}{l}49 \text { ML con EA }(22 \mathrm{~m}) \\
\text { Edad a las pruebas: } 78,82 \\
\text { Educación: } 12,31 \\
\text { RC: } 97,79 \\
\text { Estado de salud subjetivo: } 72,17 \\
\text { 37 BL con EA }(21 \mathrm{~m}) \\
\text { Edad a las pruebas: } 80,76 \\
\text { Educación: } 11,84 \\
\text { RC: } 100,45 \\
\text { Estado de salud subjetivo: } 73,5\end{array}$ & $\begin{array}{l}\text { CST de leng NART, } \\
\text { TDB (revisado), } \\
\text { Prueba Spot-the- } \\
\text { Word, BPVS, Prueba } \\
\text { de vocabulario Prawf } \\
\text { Geirfa Cymraeg i } \\
\text { Oedolion. } \\
\text { Algunos BL hablaban } \\
\text { Gal desde su } \\
\text { nacimiento y otros } \\
\text { desde los 4-5 años. } \\
\text { La edad a la cual } \\
\text { comenzaron a hablar } \\
\text { Ing variaba }\end{array}$ & $\begin{array}{l}\text { Educación ocupación, } \\
\text { situación } \\
\text { socioeconómica, RC, } \\
\text { estado de salud } \\
\text { subjetivo, ansiedad, } \\
\text { depresión, } \\
\text { medicamentos } \\
\text { inhibidores de la } \\
\text { acetilcolinesterasa }\end{array}$ & $\begin{array}{l}\text { MMSE, } \\
\text { mediciones de } \\
\text { antecedentes, } \\
\text { competencia } \\
\text { lingüística, FE }\end{array}$ & $\begin{array}{l}\text { RC similar } \\
\text { Los BL con EA } \\
\text { acudieron al servicio de } \\
\text { atención más } \\
\text { tardíamente. No hubo } \\
\text { diferencias } \\
\text { significativas entre BL } \\
\text { y ML en pruebas de } \\
\text { FE, pero los BL } \\
\text { presentaron fortalezas } \\
\text { relativas en tareas de } \\
\text { conflicto de respuesta e } \\
\text { inhibición }\end{array}$ \\
\hline
\end{tabular}




\begin{tabular}{|c|c|c|c|c|}
\hline \multicolumn{5}{|c|}{ Bialystok y otros (2014) } \\
\hline $\begin{array}{l}\text { 38 ML con DCL (19 m, } 12 \text { IN) } \\
\text { Edad de aparición: } 62,2 \\
\text { Edad clínica: } 66,5 \\
\text { Años de educación: } 15,5 \\
\text { BNA: } 95,4 \\
\text { MMSE: } 29 \\
\text { 36 BL con DCL (20 m, 25 IN) } \\
\text { Edad de aparición: } 66,9 \\
\text { Edad clínica: } 70 \\
\text { Años de educación: } 14,3 \\
\text { BNA: } 90,6 \\
\text { MMSE: } 28,4 \\
\text { 35 ML con EA (19 m, } 8 \text { IN) } \\
\text { Edad de aparición: } 70,9 \\
\text { Edad clínica: } 74,2 \\
\text { Años de educación: } 12,5 \\
\text { BNA: } 72,7 \\
\text { MMSE: } 23,4 \\
\text { 40 BL con EA (22 } \mathbf{~ m , ~ 2 7 ~ I N ) ~} \\
\text { Edad de aparición: } 78,2 \\
\text { Edad clínica: } 81,4 \\
\text { Años de educación: } 12,2 \\
\text { BNA: } 63,8 \\
\text { MMSE: } 22,3\end{array}$ & $\begin{array}{l}\text { LSBQ. } \\
\text { Todos los pacientes } \\
\text { eran competentes en } \\
\text { Ing, pero los BL } \\
\text { hablaban una } \\
\text { variedad de otras } \\
\text { leng y no } \\
\text { representaban a } \\
\text { ningún grupo } \\
\text { sociocultural } \\
\text { específico. } \\
\text { Algunos P hablaban } \\
\text { más de dos leng, pero } \\
\text { estaban incluidos en } \\
\text { el grupo de los BL }\end{array}$ & $\begin{array}{l}\text { Dieta } \\
\text { Tabaquismo } \\
\text { A. F } \\
\text { Actividad social } \\
\text { hs de INM } \\
\text { Educación }\end{array}$ & $\begin{array}{l}\text { MMSE } \\
\text { BNA } \\
3 \text { Tareas de FE } \\
\text { del D-KEFS }\end{array}$ & $\begin{array}{l}\text { Mayor RC en BL } \\
\text { Los BL mostraron } \\
\text { mayor edad en la } \\
\text { aparición de la } \\
\text { enfermedad que los ML } \\
\text { tanto para el grupo de } \\
\text { DCL (en } 4,7 \text { años) } \\
\text { como el de EA (en 7,3 } \\
\text { años) }\end{array}$ \\
\hline \multicolumn{5}{|c|}{ Bak y otros (2014) } \\
\hline $\begin{array}{l}853 \text { BL saludables }(410 \mathrm{~m} \text {, } \\
\text { aprendieron la } \mathrm{L} 2 \text { mientras } \\
\text { estudiaban) } \\
\text { Edad: } 72,49\end{array}$ & $\begin{array}{l}\text { CST administrado a } \\
\text { los P: aprendizaje de } \\
\text { cualquier L2, EdA, } \\
\text { número de leng, } \\
\text { frecuencia de uso: } \\
\text { conversación/ } \\
\text { lectura/medios de } \\
\text { comunicación } \\
\text { Sólo algunos P } \\
\text { adquirieron su L2 } \\
\text { antes de los } 11 \text { años }\end{array}$ & $\begin{array}{ll}---- \\
\end{array}$ & $\begin{array}{l}\text { Inteligencia, } \\
\text { memoria, WAIS } \\
\text { III, Prueba Moray } \\
\text { House, NART, } \\
\text { fluidez verbal }\end{array}$ & $\begin{array}{l}\text { Mayor RC en BL } \\
\text { Se observó un efecto } \\
\text { protector del } \\
\text { bilingüismo contra el } \\
\text { deterioro cognitivo } \\
\text { relacionado con la } \\
\text { edad, independiente de } \\
\text { la ii. El conocer } 3 \text { o } \\
\text { más leng produjo } \\
\text { mayores efectos que } \\
\text { conocer } 2\end{array}$ \\
\hline \multicolumn{5}{|c|}{ Woumans y otros (2015) } \\
\hline $\begin{array}{l}\text { 69 ML con EA (48 m) } \\
\text { Edad: } 76,4 \\
\text { Edad de man: } 73 \\
\text { Edad al diagnóstico: } 73,8 \\
\text { MMSE inicial: } 24,2 \\
\text { Educación: } 13,5 \\
\text { 65 BL con EA (45 m) } \\
\text { Edad: } 77,9 \\
\text { Edad de man: } 74,3 \\
\text { Edad al diagnóstico: } 75,5 \\
\text { MMSE inicial: } 23,8 \\
\text { Educación: } 14,7\end{array}$ & $\begin{array}{l}\text { Entrevistas subjetivas } \\
\text { a los pacientes y } \\
\text { cuidadores; EdA; } \\
\text { comp, uso }\end{array}$ & $\begin{array}{l}\text { Educación, ocupación } \\
\text { y situación } \\
\text { socioeconómica }\end{array}$ & $\begin{array}{l}\text { Heteroanamnesis, } \\
\text { examen físico, } \\
\text { MMSE, exámenes } \\
\text { de muestras } \\
\text { sanguíneas, } \\
\text { SPECT, TEP, TC, } \\
\text { y/oIRM }\end{array}$ & $\begin{array}{l}\text { Mayor RC en BL } \\
\text { La edad de } \\
\text { manifestación de la EA } \\
\text { fue de } 71,5 \text { en ML y } \\
76,1 \text { en BL }\end{array}$ \\
\hline
\end{tabular}




\begin{tabular}{|c|c|c|c|c|}
\hline \multicolumn{5}{|c|}{ Lawton y otros (2015) } \\
\hline $\begin{array}{l}\text { 54 ML con EA (65\% m) } \\
\text { Edad de diagnóstico de la } \\
\text { demencia: } 81,1 \\
\text { Años de educación: } 4,99 \\
\text { Puntaje 3MSE: } 78,87 \\
\text { Diabetes tipo 2: } 46 \% \\
\text { Hablantes de Esp: } 76 \% \\
\text { Hablantes de Ing: } 24 \% \\
\text { 27 BL con EA (63\% m) } \\
\text { Edad de diagnóstico de la } \\
\text { demencia: } 79,3 \\
\text { Años de educación: } 7,7 \\
\text { Puntaje 3MSE: } 79,56 \\
\text { Diabetes tipo 2: } 53 \% \\
\end{array}$ & $\begin{array}{l}2 \text { preguntas de } \\
\text { "ARSMA } \\
\text {-II" (leng/s habladas). } \\
\text { Las respuestas se } \\
\text { codificaron en una } \\
\text { escala Likert de } 0-3 \\
\text { puntos }\end{array}$ & $\begin{array}{l}\text { INM, educación, } \\
\text { diabetes }\end{array}$ & $\begin{array}{l}\text { 3MSE, } \\
\text { Test de } \\
\text { aprendizaje verbal } \\
\text { de Esp e Ing, } \\
\text { SENAS, IQCODE }\end{array}$ & $\begin{array}{l}\text { RC similar } \\
\text { Los BL tenían mejor } \\
\text { educación, pero los BL } \\
\text { nacidos en EE. UU. y } \\
\text { los ML no tenían } \\
\text { diferencias. La media } \\
\text { de la edad del } \\
\text { diagnóstico de } \\
\text { demencia fue similar en } \\
\text { ambos grupos }\end{array}$ \\
\hline \multicolumn{5}{|c|}{ Kowoll y otros (2015) } \\
\hline $\begin{array}{l}\mathbf{1 1} \text { controles BL (7 m) } \\
\text { Edad: } 68,2 \\
\text { MMSE: } 29 \\
\text { Educación: } 14,2 \\
\text { 6 controles ML (2 m) } \\
\text { Edad: } 70,2 \\
\text { MMSE: } 29,6 \\
\text { Educación: } 12,2 \\
\text { 8 BL con DCL (3 m) } \\
\text { Edad: } 71,3 \\
\text { MMSE:26,8 } \\
\text { Educación: } 13,4 \\
\text { 14 ML con DCL (5 m) } \\
\text { Edad: } 77,5 \\
\text { MMSE: } 26,4 \\
\text { Educación: } 12,2 \\
\text { 22 BL con EA (11 m) } \\
\text { Edad: } 77,2 \\
\text { MMSE: } 22 \\
\text { Educación: } 12,5 \\
\text { 25 ML con EA (9 m) } \\
\text { Edad: } 80,3 \\
\text { MMSE: } 18,9 \\
\text { Educación: } 9,5\end{array}$ & $\begin{array}{l}\text { Escala de } \\
\text { autoevaluación de 1- } \\
\text { 7; CST hs leng (EdA, } \\
\text { nivel de comp, etc. ); } \\
\text { TDB, tarea de fluidez } \\
\text { verbal de CERAD } \\
\text { Comparación entre } \\
\text { mediciones objetivas } \\
\text { y subjetivas similares } \\
\text { a las de Gollan y } \\
\text { otros (2011) }\end{array}$ & $\begin{array}{l}\text { Educación, INM, } \\
\text { dominancia } \\
\text { lingüística }\end{array}$ & $\begin{array}{l}\text { CERAD-NP, } \\
\text { MMSE, TMT, } \\
\text { Test del dibujo } \\
\text { del reloj, WMS-R } \\
\text { y WMS IV), GDS }\end{array}$ & $\begin{array}{l}\text { RC similar } \\
\text { La lg dominante puede } \\
\text { verse comprometida } \\
\text { primero en pacientes } \\
\text { bilingües con DCL. Se } \\
\text { observaron déficits de } \\
\text { la lg no dominante más } \\
\text { tarde en el curso de la } \\
\text { EA. }\end{array}$ \\
\hline
\end{tabular}

NOTAS. La tabla muestra todos los datos disponibles para cada variable en cada estudio. RC: reserva cognitiva; EA: enfermedad de Alzheimer; ML: monolingües; BL: bilingües; PL: plurilingües; m: mujeres; n: número; P: participantes; comp: competencia; man: manifestación; CST: cuestionario; con; consulta; INM: inmigración; IN: inmigrante; NIN: no-inmigrantes; A. F: actividad física; TC: tomografía computarizada; SPECT: tomografía computarizada de emisión de fotón único; L1: primera lengua, L2: segunda lengua; leng: lengua; Eval: evaluación; ii: inteligencia infantil; pres: presentación; HNI: hablante nativo de inglés; Hn. NI: hablantes no nativos de inglés; Fra: francés; Esp: español; Ing: inglés; Jap: japonés; EU: estadounidense; G: galés; Exam: examen; IA: ingreso anual; FE: función ejecutiva; MMSE: Mini examen del estado mental; 3MSE: Mini examen del estado mental modificado; IQCODE: Test del informador IQCODE; HTVL: Hopkins Verbal Learning-Test; LSBQ: Language and Social Background Questionnaire; WRAT 3: Prueba de logros con intervalo amplio; D-KEFS: Sistema de función ejecutiva 
de Delis-Kaplan; TRVS: Test de recuerdo verbal selectivo; TDB: Test de denominación de Boston; WAIS: Escala Wechsler de inteligencia para adultos; ROCF: Test de la figura compleja de Rey-Osterrieth; TMT: Test del trazo; CTT: Color Trial Test; MoCA: Evaluación cognitiva de Montreal; NART: Test nacional de lectura para adultos; Alelo e4 de APOE: Apolipoproteína E; CASI: Instrumento de evaluación de habilidades cognitivas; HAAS: Estudio de envejecimiento Honolulu-Asia; TRI: Teoría de respuesta al ítem; BIMC: Prueba de concentración-memoriainformación esperada; TRLSF: Test de recuerdo libre y selectivamente facilitado; CDR: Clasificación clínica de la demencia; ACE-R: Examen cognitivo de Addenbrooke-Revisado; DSM: Manual diagnóstico y estadístico de los trastornos mentales; TDR: Test del dibujo del reloj; Katz AVD: Actividades de la vida diaria; BNA: Evaluación de neurología conductual; GDS: escala de depresión geriátrica; BPVS: Prueba británica de vocabulario de imágenes; ARSMA: Escala de aculturación para mexicanos-americanos; SENAS: Escala de evaluación neuropsicológica español-inglés; EAC: Enfermedad de las arterias coronarias; HTN: Hipertensión; fam: familia; hs: historial; DCL: demencia con cuerpos de Lewy; DFT: demencia frontotemporal; DV: demencia vascular; ECV: enfermedad cerebrovascular; DCL amnésico: deterioro cognitivo leve amnésico. *La ocupación se determinó mediante el sistema desarrollado por el Departamento de Recursos Humanos y Desarrollo de Habilidades, Canadá (2001). Las ocupaciones se clasifican en una escala de cinco puntos, en la que los números más altos se asocian a un estatus más alto.

Recibido: 16 de agosto de 2016

Aceptado: 23 de noviembre de 2016

Publicado: 2016 